\title{
58th ANNUAL SASKATCHEWAN CHRISTMAS BIRD COUNT - 1999
}

\author{
Compiled by Wayne C. Harris, Saskatchewan Environment and Resource \\ Management, 350 Cheadle Street West, Swift Current, SK S9H 4G3
}

Christmas bird counts were conducted in 99 locations during the 1999 count period (December 16 January 3 ). This is two more counts than last year but four less than the record 103 received in 1997.

Mild temperatures and little snow cover were the norm throughout the count period. The overall average minimum temperature was only $-10^{\circ} \mathrm{C}$ and averaged $4^{\circ} \mathrm{C}$ for the high. Temperatures reached $9^{\circ} \mathrm{C}$ between Christmas and New Year's Day. The coldest temperature $\left(-34^{\circ} \mathrm{C}\right)$ was reported at Missinipe on January 2, 2000. Snow cover averaged only $5 \mathrm{~cm}$ with many southern locations reporting no snow. Winds were a factor this year with average maximum winds at 23 $\mathrm{km} / \mathrm{hr}$ making hearing birds difficult. Armit reported the highest wind speeds at $80 \mathrm{~km} / \mathrm{hr}$ although many counts had wind in excess of $30 \mathrm{~km} /$ hr (Table 1).

The number of people participating in counts was 766 . They covered over $600 \mathrm{~km}$ on foot in 350 hours, travelled over $13,000 \mathrm{~km}$ by vehicle in 650 hours, all well above average (Table 2).

\section{The birds}

One hundred and twelve species were reported this year and all but four of these were on count day. In addition to these there were two recognizable subspecies and one hybrid seen (Tables
3,4 and 5). This is the second highest species total ever reported and is only the fourth time 100 or more species have been reported.

The highest number of species seen on a count was 42 at Coronach although Saskatoon, with 40 count day species, plus four count period species, was the highest overall. Another 10 counts exceeded 30 species, making this one of the best years ever for species diversity on individual counts.

The number of individual birds seen exceeded 145,000, the second highest total ever. Mallard was the most abundant bird and with 24 localities reporting the species, provided further evidence of the mild weather during the fall and early winter. Not surprisingly, Snow Bunting was the second most abundant, although this total was bolstered by a couple of large counts, making the total deceiving. In general, Snow Buntings were scarce and only ranked $9^{\text {th }}$ on the frequency list instead of the usual first or second. Third on the list of abundance was the Common Redpoll with over 19,000 individuals reported, but even more surprising was the fact that they were recorded on $95 \%$ of the counts, making them the most frequently occurring species on counts, outranking both Black-billed Magpie and House Sparrow. 


\section{New Species}

Four new species were reported this year bringing the provincial Christmas bird count total to 172 species. The first was a Ferruginous Hawk on the Leader South count. This species normally goes south to Texas for the winter, but this year, with the warm weather, ground squirrels remained active and a few Ferruginous Hawks remained here later than normal.

The second new species was Eurasian Collared-Dove. A pair of this species was found in each of Regina and Weyburn. In each case, the pair of birds had arrived earlier in the year and had taken up residence at a feeder. The Eurasian Collared-Dove arrived in North America in the early 1970 s with an introduction to the Bahamas, spreading from there to the Florida Keys and then north to Florida. Populations have established in Florida, Alabama and Texas. From these established populations, pairs of juvenile birds are apparently under-taking long distance northern movements to such areas as Wisconsin, Michigan, Minnesota, Montana and now Saskatchewan. It will be interesting to see if they successfully overwinter and breed here.

The next new species is appropriately named but still well out of its normal winter range - a Winter Wren. This bird was found foraging in a brushy area along Battle Creek in the Cypress Hills and is Saskatchewan's first winter record.

Last is a Brambling which arrived at Hazel and Myron Barton's feeder at Macdowall on December 31 and stayed for three days before moving on. This bird was associating with a group of redpolls. This is an Asian species which shows up occasionally as a straggler along the Pacific coast mostly in Alaska although there are records for both
Manitoba and Montana. This is the second Saskatchewan record, the first being from Saskatoon in 1991.

\section{Other rarities}

Table 4 lists the least frequent species on the counts and the rarest species are all in this table. The second count and first count day record of a Field Sparrow was one at a Birch Hills feeder; the only other count record was a count period bird from Saskatoon in 1985. A Harlan's Hawk on the Fort Walsh count was only the third record; the first was at White Bear in 1995 and the second at Govenlock in 1997.

\section{Population trends}

A number of new high counts were recorded (Table 6). Most notable, and following the trend discussed last year was the 247 House Finches at Saskatoon which exceeded the 190 in Regina last year. The 10 American Goldfinches at Craven was one more than last year's record 9 at Kenosee Lake.

Waterfowl numbers were well above the normal with both Canada Geese and Mallard showing large increases (Table 7). Northern Goshawk numbers were down as were Merlin. Although Ruffed Grouse numbers were down from last year they were above the long term average. Spruce Grouse showed a large increase over last year and were well above the long term average.

Several irruptive species appeared in above normal numbers. Red-breasted Nuthatches were well above normal but it was the redpolls that put on the best show. Numbers of both Common and Hoary Redpolls were two to three times the normal. Yet other species often associated with these winter irruptions were conspicuously uncommon, in particular Pine Grosbeaks and both crossbill species. 
Count areas and Participants

(Names of compilers are in italics).

1.ARCHERWILL. Fay Black, Ken Folstad, Carmel Fountain, Judy Revoy, Doris Slind.

2.ARMIT. Valeri Harris, Wayne Harris, Sheila Lamont, Bill Zak, Joyce Zak.

3. ASSINIBOIA. Ed Bearss, Jack Burgeson, Cecil Hayward, David Landa, Delmer Pettem, Wilf Prentice, Ken Schuweiler.

4.AVONLEA - SPRING VALLEY. Larry Bogdan.

5.BANGOR. Allan Bolton, Lynn Bolton, Judith Davis, Aileen Hayward, Jean Hilton, Minnie Hughes, Bradley Thompson, Norman Thompson.

6.BEAUVAL.Clara Sanderson, Rick Sanderson.

7.BIGGAR. Dale Booth, Guy Wapple, Marguerite Wapple, Robert Wapple, Sandra Wapple.

8.BIG RIVER. Colleen Honig, Glenn Honig.

9.BIRCH HILLS. Carman Dodge, Margaret Mareschal, Moe Mareschal, Don Weidl.

10.BODMIN. Carla Braidek.

11.BROADVIEW. David Chasckavich, Don Weidl, Tony Weidl.

12.BROMHEAD. Martin Bailey, Carol Bjorklund.

13.CABRI. Carman Dodge.

14. CANDLE LAKE. Carman Dodge, Dave Stepnisky, Lisa Takats, Don Weidl.
15.CLARK'S CROSSING. Lucille Bradatsch, Donna Bruce, Melanie Elliott, Witek Gierulski, Marilyn Haskins, Jim Hay, Robert Johanson, Marlene Kalanack, Richard Kerbes, Gordon Koshinsky, Margaret Koshinsky, Gerard Lahey, Janet McVittie, Hilda Noton, Lynn Oliphant, Keith Pahl, Betty Penner, Stan Shadick, Rhonda Shewfelt, Wayne Sheils, Eli-Ann Stoffel, Martin Stoffel, Kirk Wallace, Michael Williams, Jim Wood.

16.COCHIN. Daryl Johannesen, Kaitlin Johannesen, Kristine Johannesen.

\section{CORONACH. Wayne Harris.}

18. CRAVEN. Mary Aird, Margaret Belcher, Betty Binnie, David Cowley, Jim Cummings, Karen Elliott, Trevor Herriot, Louise Holloway, Phil Holloway, Bob Kreba, Kevin Moore, Ron Myers, Bill Ogilvie.

19. CROOKED LAKE. Bill Livesay, Mayta Livesay, Boyd Metzler, John Pollock, Dorothy Skene, Ed Skene.

20.CROOKED RIVER. Margaret Mehler, Morley Mehler.

21.CYPRESS HILLS PROVINCIAL PARK (Centre Block). Gary Churchill, John Douglas, Shirley Helmerson, Marilyn Scheller, Wayne Harris, Joan Hodgins, Jeff Jensen, Ron Jensen, Harvey Johnson, Cameron Lockerbie, Melody Nagel-Hisey.

22.DUCK LAKE. Keith Hobson, Alan Smith.

23.EASTEND. Evelyn Brown, Betty Davis, Robert Gebhardt, Joan Hodgins, Harvey Johnson.

24. EMMA LAKE. Glen Hanson, Jean Hanson, Deanna Krug, Norman Krug. 
25. ESTEVAN. Marlis Buck, Wayne Harris, Sheila Lamont.

26. ESTUARY NORTH. Barry Cocks, Cathy Cocks, Chris Francis, Dean Francis, Frances Hartsook.

27.FENTON. Carman Dodge, Don Weidl.

28.FERLAND. Don Davidson, Jacqueline Davidson, Martha Davidson, Steven Gauthier, Cameron Jackson.

29.FIFE LAKE. Martin Myers, Robert Rafuse.

30.FORT QU'APPELLE. James Armstrong, Phyllis Bordass, Anne Davies, Ida Grosse, Doreen Harman, Ronald Hooper, Alice Isfan, Keith James, Lois Lamontagne, Vic Lamontagne, Allan Lewis, John Lowe, Don McDougall, Jean McKenna, Allan Mlazgar, Delores Palmer, Webb Palmer, Paul Paquin, Lorne Rowell, Fred Warren.

31.FORT WALSH. Pat Bulman, Valeri Harris, Wayne Harris, Al Hartley, Ron Jensen, Sheila Lamont, Wilkes Parsonage, Wayne Renaud, Guy Wapple.

32.GARDINER DAM. Pat Bulman, Greg Fenty, Valeri Harris, Wayne Harris, Al Hartley, Mary Houston, Stuart Houston, Sheila Lamont, Cameron Lockerbie, Dennis Pederson, Wayne Renaud, Martin Stoffel, Guy Wapple, Dan Zazelenchuk.

33.GOOD SPIRIT LAKE. Bill Anaka, Joyce Anaka, Dorothy Riesz, Ray Riesz, Julia Wiwchar.

34.GOVENLOCK. Pat Bulman, Valeri Harris, Wayne Harris, Sheila Lamont, Wayne Renaud, Guy Wapple.
35. GRASSLANDS NATIONAL PARK (NW). Roy Cote, Pat Fargey, Laura James, Dennis Madsen, Lorie Wiesner, Collette Schmidt, Adrian Sturch.

36. GRAYSON. Charles Helm, Daniel Helm, Karl Zimmer.

37.HARRIS. Guy Wapple, Robert Wapple.

38. HEPBURN. Helen Fehr, Phyllis Siemens.

39. HOLBIEN. Chlorus Harris, Helen Harris.

40.INDIAN HEAD. Jan Beattie, Carol Beaulieu, Denise Beaulieu, Margot Beaulieu, Rhys Bealieu, Vic Beaulieu, Chris Blair, Irv Escott, M. Escott, S. Escott, David Gehl, Mavis Gray, Gordon Howe, Dora Nichols, Brian Scott, Lorne Scott, Heidi Scott, Ann Willerth, Gordon Willerth.

41.KAMSACK. John Barisoff, George Bernhard, Agnes Betz, Marlon Brock, Mable Buceuk, Fred Chernoff, Jeff Conley, Lindee Dewores, Judy Dubasov, Bill Koroluk, Adeline Nykolaishen, Molly Poole, Kelsey and Ryley Rezansoff, Isabel Ritchie, Metro Rosowsky, Wally Sasyniuk, Elsie Severson, Don Smandych, Arlene Smorodin, Elenor Sookocheef, Pete Unlow.

42. KELVINGTON. Dianne Sloan, Marguerite Sloan.

43.KENASTON. Lawrence Beckie.

44.KENNEDY. Glen Heebner, Boyd Metzler

45.KENOSEE LAKE. Boyd Metzler, John Pollock.

46.KILWINNING. Ed Driver, AI Smith 
47. KINDERSLEY. Jean Harris, Keith Harris.

48. KINLOCH. Don Forbes, Doreen Forbes, Merle Jackson, Cliff Logan, Wilf Rodenberg, Doreen Wickstrom.

49.KUTAWAGAN LAKE. Wayne Harris, Sheila Lamont, Allan Rahn.

50.LAIRD. Alice Nickel, Arley Nickel

51.LAST MOUNTAIN LAKE N.W.A. Leland Greenfield, Valeri Harris, Wayne Harris, Sheila Lamont.

52.LEADER (North). Daisy Meyers.

53.LEADER (South). Brenda Flood, John Flood.

54.LIVELONG. Sarah Pavka.

55.LOVE - TORCH RIVER. Ken Blaine, Lorna Blaine, Bert Dalziel, Duke Dalziel, Joan Dalziel, Kari Dalziel, Nora Dalziel, Sara Dalziel, Betty Donovan, Bruce Donovan, Eileen L'Heureux, Paul L'Heureux, Lynn Matthews, William Matthews.

56.LUSELAND. Doug Campbell, Estelle Finley, Kim Finley, Sandra Finley, Jean Halliday, Brent Honeker.

57.MACDOWALL. Hazel Barton, Myron Barton.

58.MATADOR. Cindy Romo, Jim Romo.

59.MEADOW LAKE. Bill Caldwell, Betty Ann Golly, Byron Golly, Stuart Golly, Tyler Golly, Alexes Lockwood, Pat Sampson, Brenda Twidale, Ian Twidale, Lorraine Twidale, Joe Twidale, John Weir, Bob Wilson.

60.MELFORT. Frieda Markland.
61.MELVILLE. Donna Alberts, Marion MacLean, Mark Shepherd, Ross Wotherspoon.

62.MISSINIPE. Shirley Glass.

63.MOOSE JAW. Elsie Adkins, Jack Adkins, Doug Brunsdon, Helen Brunsdon, Barry Dowse, Fern Dowse, Al Gurnsey, Terry Gurnsey, Trevor Gurnsey, Kerry Hanley, Robert Hanley, Carol Howes, Ralph Howes, Eve King, Cy Knight, Leith Knight, Allan Lemieux, Shirley Lemieux, Bill McDonald, Jean Anne Mowchenko, Len Mowchenko, Helen Norys, Peter Norys, Wilma Pickering, Ramsay Ross, Gus Sagall, Mike Sagall, Lloyd Stewart, Brenda Winch.

64.MOOSE MOUNTAIN. Greg Bobbitt, Dick Gutfriend, Keith Sakatch, Stewart Stairmand.

65. NIPAWIN. Inge Brown, Vi Budd, Joyce Christiansen, Bert Dalziel, Bruce Donovan, Bryan Friswell, Judith Friswell, Betty Hodgins, Hildegard Lessing, Doug Pegg, Helen Pegg, Shirley Phillips.

66. NISBET FOREST WEST (NW of Crutwell). Kim Clark, Kiri Clark, Shamara Clark, Suzanne Clark, Evelyn Marshall.

67.PIKE LAKE. Tony Allen, Lawrence Beckie, Kurt Brauner, Donna Bruce, Ann Christiansen, Pat Christiansen, David Cook, Louise Cook, Jill Cornford, Peter Cornford, Bob Girvan, Bernie Gollop, Mike Gollop, Don Johnston, Shirley Johnston, Elaine Konkin, Fred Konkin, Kay Krueger, Donna Nazar, Meno Nickel, Bruce Noton, Hilda Noton, Keith Pahl, Frank Roy, Stan Shadick, Jan Solem, Angela Stern, Martin Stoffel, Michael Williams, Michael Willson, Phil Willson. 
68.PORCUPINE PLAIN. Agnes Hodge, Keith Larwood, Pat Larwood, Phyllis Swaan.

69.PREECEVILLE. Mary Mitchell, Muriel Mitchell, Mae Tunbridge.

70.PADDOCKWOOD. Barry Day, Alfred Corriveau, John Dinius, Serg McGowan, John Toporowski

71.PRINCE ALBERT. John Burt, Pamela Burt, Jackie Carter, Steve Carter, Carman Dodge, Keith Dodge, Ken Dodge, Robert Fahlman, Joan Firth, Millie Hjertaas, Sandra Jewel, David Junor, Murray Little, Penny Mindiuk, Joan Pereverzoff, Helen Placsko, Joe Placsko, Fanny Prins, Christine Rye, Deborah Rye, John Rye, Michael Rye, Peter Rye, Les Senner, Winona Senner, Katherine Sutton, Shirley Swain, Ollie Thoresen, Nancy Vizina, Don Weidl.

72.PRINCE ALBERT NATIONAL PARK. Susan Carr, Richard Cherepak, Yolande Cherepak, Dan Frandsen, Brad Lloyd, Paula Pacholek, Cam Zimmer.

73.QU'APPELLE VALLEY DAM. Witek Gierulski, Stan Shadick, Michael Williams.

74.RAYMORE. Valeri Harris, Wayne Harris, Sheila Lamont.

75.REGINA. Ross Barclay, Gladys Barr, Keith Barr, Lionel Bonneville, Stephane Bonneville, Jim Elliott, Shirley Gerlock, Isabelle Girard, Trevor Herriot, Dale Hjertaas, Estelle Hjertaas, Kerry Hjertaas, Paule Hjertaas, Phyllis Ilsley, Winnifred Ilsley, Bob Kreba, Linda Langenbacher, Rheal Laroche, Yvonne McKenzie, Ron Meyers, Catrina Ottosin, Charles Ottosin, Diane Ottosin, Jean Ottosin, Catherine Parkinson, Wayne Pepper, Brian Rainey, Curt Schroeder, Diane Secoy, Frank Switzer, May Switzer, Audrey Tranquair, Ian Tranquair.
76.ROCKGLEN - BORDERLAND. Bob Rafuse, Martin Meyers.

77.ROUND LAKE (Qu'Appelle Valley). Pat Connolley, Doug Francis, Boyd Metzler, John Pollock.

78.SASKATCHEWAN LANDING PROVINCIAL PARK. Calvin Fiala, Wayne Harris, Ron Jensen, Cameron Jordheim, Sig Jordheim, Cameron Lockerbie.

79.SASKATCHEWAN RIVER FORKS. Carman Dodge, Don Weidl.

80.SASKATOON. Bruce Acton, Don Adams, Darlene Aikman, Nancy Allan, Tony Allen, Juhachi Asai, Sumiko Asai, Don Beazely, Margaret Belcher, Bob Besant, Jeff Besant, Joyce Besant, Bruce Brotzel,Doris Bietenholz, Eveline Boudreau, Gail Bunt, Rick Bunt, David Cook, Louise Cook, Paul Coutu, Vi Coutu, Ann Coxworth, Ewen Coxworth, Leah Currie, Eileen Dalglish, Ed Driver, Lorne Duczek, Brian Duggan, Ann Dzus, Randi Edmonds, Fran Eldridge, Melanie Elliott, Helen Fast, Ken Froese, Mary Gilliland, Cedric Gillott, Bernie Gollop, Maddie Gollop, Mike Gollop, Jean Gordon, Bob Green, Jerry Haigh, Bruce Hanbidge, Helen Hanbidge, John Hanbidge, Kaija Harris, Tim Haughian, Jim Hay, John Horton, Mary Houston, Stan Houston, Stuart Houston, Kathryn Hull, Don Johnston, Marlene Kalanack, Richard Kerbes, Kay Klassen, Elain Konkin, Fred Konkin, Gordon Koshinsky, Barbara Kozmyk, Gerard Lahey, Harvey Lane, Catherine Laratte, Erling Larsen, Grethe Larsen, Anna Leighton, Ted Leighton, Cameron Lockerbie, Bill MacKenzie, Catherine Madore, Edna McCawley, Terry McCawley, Cleve Mellor, Cathryn Miller, David Miller, Muriel Miller, Garth Nelson, Lillian Nelson, Menno Nickel, Bruce Noton, Hilda Noton, Randy Olson, Keith Pahl, Amanda Plante, Garry Rector, 
Allan Rosenberg, Marella Rosta, Andy Salisbury, Craig Salisbury, Lorriene Salisbury, Rob Salisbury, Maureen Scarfe, Phil Scotland, Susan Skinner, Jim Smart, Al Smith, Angela Stern, Martin Stoffel, Phil Taylor, Hilda Voth, Heather Wagg, Kirk Wallace, Shirley Wedgwood, George West, Colette Wheler, Gordon Wilkinson, Michael Williams, Jennifer Willson, Kay Willson, Phil Willson, Jim Wood, Lois Wooding, David Wright, Norman Zlotkin.

81.SHAMROCK. Donna Heinrichs, Jaimee Heinrichs, Sharon Heinrichs, Hugh Henry.

82.SKULL CREEK. Catherin Bennetto, Eileen Bennetto, Ray Bennetto, Tim Bennetto, Bob Nyen, Derek Nyen, Vivian Nyen.

83.SNOWDEN. Bonny Bailey, Elenor Bodnaruk, Mike Bodnaruk, Bert Dalziel, Kari Dalziel, Sara Dalziel, Diane Friesen, Don Friesen, Irene Hagel, Karen Hagel, Ben Holmquist, Lily Kuzniar, Bob Olson, Janet Olson, Jack Pickett, Ole Rudd, Vera Schemenauer, Orville Shwetz, Vicky Shwetz, Bev Smears, Walter Stevenson, Bernice Tornquist, Torance Torquist.

84.SOMME. Edwin Billeter, Sharon Birch, Wesley Black, Irene Brehmer, Florence Chase, Margaret Hooper, Ronald Hooper, Gordon Johnson, Elsie Kerr.

85.SPALDING. Olwen Hoffman, Dora Knutson, Virginia Perron, Bill Spizawka, Velma Spizawka.

86.SQUAW RAPIDS. Pat Bulman, Valeri Harris, Wayne Harris, Sheila Lamont, Guy Wapple.

87.STOCKHOLM. Douglas Flatt, Gordon Smith.
88.SWIFT. CURRENT. Barbara Adam, Evelyn Anderson', James Beattie, Jackie Bolton, Merl Campion, Tracy Chanig, Noris Currie, Laurent Dudragne, Mary Ann Dudragne, Michelle Dudragne, Hugh Henry, Jeff Jensen, Ron Jensen, Doreen Kreuger, Walt Kreuger, Arlene Lawson, Doug Lawson, Cameron Lockerbie, Kimberly Marsden, Eloise Miller, Max Miller, Francis Moen, Ed Nein, Ted Philipchuk, Ernest Schlickting, Jeanne Schlickting, Irene Stinson, Doris Thoreson, Doris M. Thoreson, Betty Welgan, Ron Welgan, John Weston, Pearl Weston, Verdella Zacharias.

89.TISDALE. Carl Mohr, Joyce Mohr, Percy Yelland.

\section{TOGO. Barb Elsasser, Doug Elsasser.}

91.TORCH RIVER VALLEY. Bert Dalziel, Bruce Donovan, George Lidster, Jean Lidster, Dale Nelson, Jeanette Nelson, Curtis Riemer.

92.TURTLE LAKE. E.M. Robinson, Marie Robinson.

93.WALDHEIM. Bailey Epp, Barb Epp, Murray Epp, Lorena Janzen, Wimpy Janzen.

94.WATSON.Doreen MacDonald, Irene Sampson, Patricia Sterzuk, Doreen Weber.

95.WELDON. Ada Hawrish, Bernard Hawrish.

96. WEYBURN. Leo Belanger, Jim Burge, Bob Cameron, Lucille Cameron, Fred Garner, Lynne Garner, George Gillies, Doug Goranson, Mavis Goranson, Dick Gutfriend, Betty Layh, Phil Layh, Cyril Marcotte, Alma McCormick, Keith Sakatch, Stewart Stairmand, Joe Weisgerber, Rose Weisgerber. 
97. WHITE BEAR. Darryl Jordheim, Floyd Jordheim, Laine Jordheim, Sig Jordheim, Elijah MacCulloch, Greg MacCullock, Lynette MacCullock.

98.WHITEWOOD. Ellen Blyth, Stephen Ede, Tom Ede, Wilfred Jordens, Bernice Juzyniec, Felix Juzyniec, Joyce Kydd, Jean Meadows, Boyd Metzler, John Pollock, Alfred Pritchard, Florence
Pritchard, Lenore Santo, Diane Veresh, Pat Ward, Jean Wilson, Edwin Wyatt, Elaine Wyatt.

99.YORKTON. Bill Anaka, Joyce Anaka, Elinor Hjertaas, Lance Irving, Mark Kornder, Boyd Metzlar, Ray Riesz, Geoff Rushowick, Patrick Rushowick, Tesa Rushowick, Dorothy Skene, Harold Wilkinson, Wilma Wilkinson.
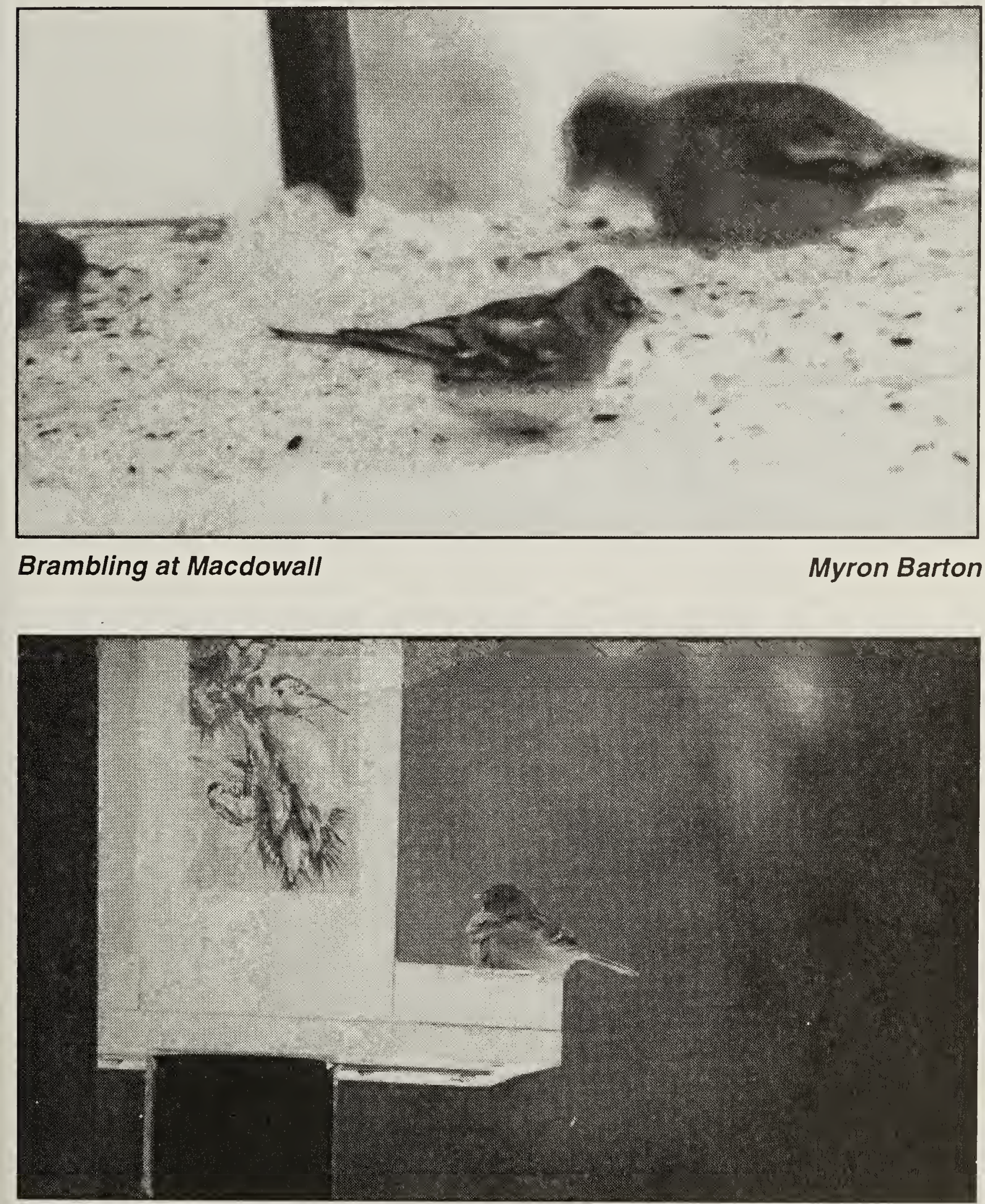
Figure 1. Location of 1999 counts (numbers correspond to locality names in Tables)

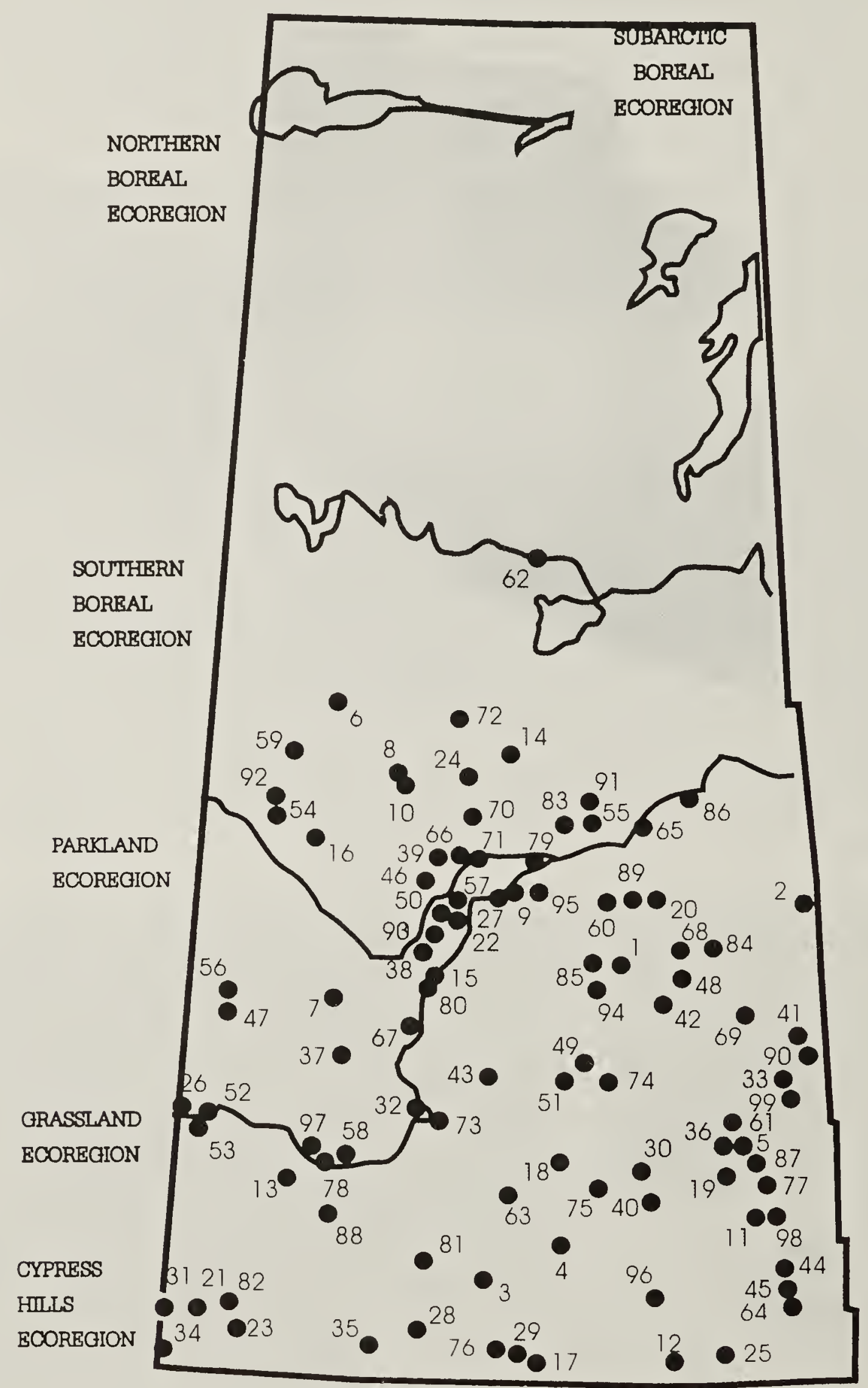




\begin{tabular}{|c|c|c|c|c|c|c|c|c|c|}
\hline COUNT & DATE & 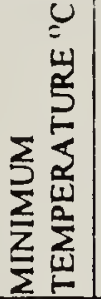 & 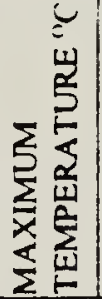 & 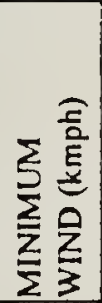 & 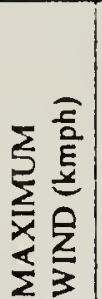 & 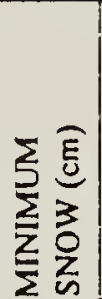 & 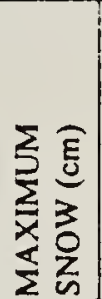 & SKY AM & SKY PM \\
\hline ARCHERWILL & Dec. 30 & -28 & -24 & 5 & 15 & 1 & 2 & light snow & light snow \\
\hline ARMIT & Dec. 27 & -11 & 2 & 30 & 80 & 5 & 20 & partly & partly \\
\hline ASSINIBOIA & Jan. 03 & 0 & 2 & 25 & 35 & 5 & 8 & partly & partly \\
\hline AVONLEA & Jan. 02 & -19 & -18 & 0 & 5 & 7 & 10 & & light fog \\
\hline 5 BANGOR & Dec. 17 & -15 & -3 & 5 & 10 & 3 & 10 & partly & overcast \\
\hline 6 BEAUVAL & Dec. 26 & -6 & -1 & 0 & 10 & & 40 & partly & mostly clear \\
\hline BIGGAR & Dec. 27 & -2 & 6 & 20 & 60 & 0 & 10 & partly & partly \\
\hline BIG RIVER & Jan. 02 & -22 & -22 & 0 & 0 & 30 & 40 & & \\
\hline BIRCH HILLS & Dec. 20 & -26 & -15 & 5 & 20 & 2 & 40 & clear & overcast \\
\hline 10 BODMIN & Dec. 24 & 0 & 4 & 0 & 0 & 4 & 10 & clear & \\
\hline 11 BROADVIEW & Dec. 26 & -3 & 2 & 15 & 30 & 0 & 2 & overcast & partly \\
\hline 12 BROMHEAD & Jan. 02 & -8 & -6 & 10 & 15 & 0 & 1 & overcast & overcast \\
\hline 13 CABRI & Dec. 25 & -5 & 9 & 5 & 10 & 0 & 2 & partly & mostly clear \\
\hline 14 CANDLE LAKE & Dec. 30 & -7 & -4 & 20 & 25 & 10 & 15 & & \\
\hline 15 CLARK'S CROSS. & Dec. 18 & -9 & -3 & 15 & 35 & 2 & 10 & overcast & overcast \\
\hline $16 \mathrm{COCHIN}$ & Dec. 27 & 2 & 6 & 0 & 5 & 10 & 25 & partly & partly \\
\hline 17 CORONACH & Dec. 22 & -8 & 0 & 10 & 50 & 0 & 8 & light fog & light fog \\
\hline 18 CRAVEN & Dec. 18 & -4 & -2 & 20 & 30 & 0 & 8 & mostly clear & partly \\
\hline 19 CROOKED LAKE & Dec. 18 & -8 & -1 & 5 & 30 & 3 & 5 & overcast & overcast \\
\hline 20 CROOKED RIVER & Dec. 30 & -20 & -20 & 10 & 20 & & & light fog & light fog \\
\hline 21 CYPRESS HILLS & Dec. 30 & -2 & 1 & 0 & 10 & 0 & 15 & clear & mostly clear \\
\hline 22 DUCK LAKE & Dec. 19 & -20 & -15 & 10 & 20 & 10 & 30 & clear & clear \\
\hline 23 EASTEND & Dec. 27 & -1 & 8 & 0 & 35 & 0 & 0 & clear & clear \\
\hline 24 EMMA LAKE & Jan. 01 & -18 & -16 & 5 & 15 & 30 & 45 & mod. snow & mod. snow \\
\hline 25 ESTEVAN & Dec. 23 & -7 & 2 & 10 & 40 & 0 & 5 & mostly clear & mostly clear \\
\hline 26 ESTUARY & Dec. 31 & -7 & -3 & 0 & 5 & 0 & 3 & partly & partly \\
\hline 27 FENTON & Dec. 18 & -9 & -9 & 20 & 28 & 2 & 5 & overcast & light snow \\
\hline 28 FERLAND & Dec. 30 & 1 & 8 & 0 & 15 & 0 & 0 & mostly clear & clear \\
\hline 29 FIFE LAKE & Dec. 28 & 4 & 7 & 5 & 25 & 0 & 0 & clear & clear \\
\hline 30 FT. QU'APPELLE & Dec. 18 & -6 & -4 & 20 & 22 & 3 & 4 & overcast & overcast \\
\hline 3I FORT WALSH & Dec. 18 & -3 & 4 & 10 & 70 & 0 & 5 & light rain & light snow \\
\hline 32 GARDINER DAM & Dec. 20 & -31 & -18 & 10 & 30 & 0 & 5 & partly & partly \\
\hline 33 GOOD SPIRIT & Dec. 29 & -3 & 5 & 15 & 25 & 0 & 5 & partly & partly \\
\hline 34 GOVENLOCK & Dec. 19 & -17 & -15 & 30 & 70 & 0 & 0 & mostly clear & partly \\
\hline 35 GRASSLANDS NP & Dec. 17 & -10 & 4 & 4 & 22 & 0 & 1 & clear & clear \\
\hline 36 GRAYSON & Dec. 23 & -5 & 1 & 0 & 10 & 0 & 10 & mostly clear & mostly clear \\
\hline 37 HARRIS & Jan. 03 & -28 & -21 & 0 & 20 & 0 & 8 & partly & partly \\
\hline 38 HEPBURN & Dec. 29 & 2 & 5 & 0 & 0 & 4 & 8 & clear & mostly clear \\
\hline 39 HOLBEIN & Dec. 31 & -20 & -15 & 25 & 30 & 10 & 15 & clear & partly \\
\hline 40 INDIAN HEAD & Dec. 27 & -10 & 6 & 0 & 40 & 0 & 5 & & clear \\
\hline $41 \mathrm{KAMSACK}$ & Dec. 26 & & & 10 & 20 & 10 & 15 & clear & \\
\hline 42 KELVINGTON & Dec. 28 & -1 & 3 & 5 & 10 & 8 & 10 & clear & clear \\
\hline 43 KENASTON & Dec. 23 & -9 & 0 & 0 & 10 & 5 & 10 & clear & clear \\
\hline 44 KENNEDY & Dec. 30 & -5 & -2 & 20 & 50 & 0 & 3 & partly & clear \\
\hline 45 KENOSEE LAKE & Dec. 28 & 2 & 6 & 10 & 40 & 0 & 2 & mostly clear & partly \\
\hline 46 KILWINNING & Dec. 22 & -13 & -2 & 0 & 0 & 10 & 20 & mostly clear & mostly clear \\
\hline 47 KINDERSLEY & Dec. 27 & 0 & 4 & 10 & 15 & 0 & 3 & clear & clear \\
\hline 48 KINLOCH & Dec. 18 & -10 & -4 & 10 & 20 & 5 & 8 & overcast & overcast \\
\hline 49 KUTAWAGAN L. & Dec. 24 & -5 & 3 & 10 & 30 & 2 & 10 & clear & clear \\
\hline 50 LAlRD & Dec. 28 & & & & & 10 & 20 & partly & clear \\
\hline
\end{tabular}




\begin{tabular}{|c|c|c|c|c|c|c|c|c|c|}
\hline COUNT & DATE & 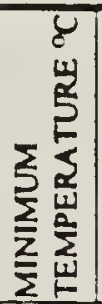 & 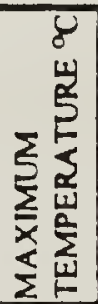 & $\sum_{\sum}^{\sum}$ & 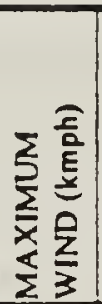 & 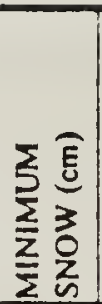 & 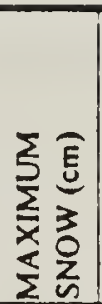 & SKY AM & SKY PM \\
\hline 51 LAST MT. LAKE & Dec. 26 & -9 & 4 & 30 & 50 & 2 & 6 & partly & partly \\
\hline 52 LEADER $(\mathbb{N})$ & Dec. 27 & -4 & 9 & 0 & 30 & 0 & I & clear & clear \\
\hline 53 LEADER (S) & Dec. 18 & 0 & 5 & 5 & 10 & 0 & 0 & overcast & overcast \\
\hline 54 LIVELONG & Dec. 21 & -20 & -15 & & & 2 & & mostly clear & mostly clear \\
\hline 55 LOVE & Dec. 24 & -2 & -2 & 10 & 25 & 20 & 28 & clear & clear \\
\hline 56 LUSELAND & Dec. 26 & 2 & 6 & 0 & 10 & 0 & 5 & mostly clear & mostly clear \\
\hline 57 MACDOWALL & Dec. 25 & -3 & 4 & 30 & 40 & 10 & 15 & clear & clear \\
\hline 58 MATADOR & Dec. 27 & 0 & 9 & 5 & 35 & 6 & 12 & mostly clear & clear \\
\hline 59 MEADOW LAKE & Dec. 26 & 0 & 3 & 0 & 10 & 0 & 20 & clear & clear \\
\hline 60 MELFORT & Dec. 26 & & & & & & & & \\
\hline 61 MELVILLE & Dec. 25 & -8 & 3 & 25 & 50 & 0 & 10 & mostly clear & overcast \\
\hline 62 MISSINIPE & Jan. 02 & -34 & -30 & 0 & 0 & 50 & 50 & mostly clear & mostly clear \\
\hline 63 MOOSE JAW & Dec. 26 & -7 & 5 & 15 & 30 & 0 & 0 & mostly clear & mostly clear \\
\hline 64 MOOSE MTN. & Dec. 30 & -4 & -1 & 5 & 35 & 0 & 3 & overcast & mostly clear \\
\hline 65 NIPAWIN & Dec. 30 & -13 & -13 & 5 & 10 & 6 & 25 & light snow & light snow \\
\hline 66 NISBET FOREST & Jan. 01 & -22 & -15 & 5 & 10 & 20 & 30 & light snow & light snow \\
\hline 67 PIKE LAKE & Jan. 02 & -24 & -20 & 5 & 15 & 5 & 15 & light snow & light snow \\
\hline 68 PORCUPINE PLN. & Dec. 27 & -10 & 1 & 10 & 15 & 10 & 15 & clear & clear \\
\hline 69 PREECEVILLE & Jan. 01 & -21 & -21 & 15 & 20 & 10 & 15 & light snow & light snow \\
\hline 70 PADDOCKWOOD & Dec. 26 & & & & & & & & \\
\hline 71 PRINCE ALBERT & Dec. 19 & -25 & -20 & 20 & 40 & 10 & 70 & clear & clear \\
\hline 72 PR. ALBERT NP & Dec. 19 & -15 & -10 & 10 & 15 & & & & \\
\hline 73 QU'APPELLE DM & Dec. 19 & -25 & -20 & 30 & 60 & 0 & 6 & mostly clear & mostly clear \\
\hline 74 RAYMORE & Dec. 25 & 0 & 4 & 30 & 50 & 5 & 15 & mostly clear & clear \\
\hline 75 REGINA & Dec. 26 & -1 & 3 & 10 & 25 & 0 & 0 & mostly clear & mostly clear \\
\hline 76 ROCKGLEN & Dec. 30 & -3 & -1 & 0 & 0 & 0 & 0 & clear & clear \\
\hline 77 ROUND LAKE & Dec. 20 & -28 & -20 & 10 & 20 & 0 & 5 & clear & clear \\
\hline 78 SK. LANDING PP & Dec. 21 & -12 & -5 & 5 & 15 & 0 & 15 & mostly clear & partly \\
\hline 79 SASK. R. FORKS & Dec. 20 & -19 & -18 & 0 & 10 & 5 & 15 & overcast & overcast \\
\hline 80 SASKATOON & Dec. 26 & -5 & 4 & 10 & 30 & 3 & 15 & partly & partly \\
\hline 81 SHAMROCK & Dec. 29 & 3 & 6 & 5 & 15 & 0 & 15 & mostly clear & mostly clear \\
\hline 82 SKULL CREEK & Dec. 26 & -5 & 0 & 5 & 20 & 0 & 1 & partly & partly \\
\hline 83 SNOWDEN & Dec. 27 & 2 & 8 & 0 & 0 & 2 & 6 & overcast & mostly clear \\
\hline 84 SOMME & Dec. 27 & -2 & 6 & 30 & 60 & 7 & 10 & clear & clear \\
\hline 85 SPALDING & Dec. 27 & -10 & 7 & 30 & 50 & 6 & 10 & overcast & clear \\
\hline 86 SQUAW RAPIDS & Dec. 28 & 0 & 5 & 5 & 10 & 20 & 40 & partly & overcast \\
\hline 87 STOCKHOLM & Dec. 28 & -8 & 7 & & & 0 & 2 & partly & partly \\
\hline 88 SWIFT CURRENT & Jan. 02 & -18 & -12 & 5 & 9 & 2 & 10 & light snow & mod. snow \\
\hline 89 TISDALE & Dec. 24 & -6 & -2 & & & 10 & 12 & partly & overcast \\
\hline 90 TOGO & Dec. 24 & -15 & -8 & 40 & 50 & 2 & 5 & partly & partly \\
\hline 91 TORCH RIVER & Jan. 02 & -33 & -25 & 0 & 15 & 30 & 40 & & \\
\hline 92 TURTLE LAKE & Dec. 20 & -15 & -10 & 5 & 10 & 12 & 12 & overcast & partly \\
\hline 93 WALDHEIM & Dec. 30 & -5 & -3 & 5 & 7 & 3 & 30 & & clear \\
\hline 94 WATSON & Jan. 02 & -22 & -18 & 0 & 8 & 10 & 15 & mostly clear & mostly clear \\
\hline 95 WELDON & Dec. 19 & -30 & -25 & 30 & 50 & 15 & 18 & partly & mostly clear \\
\hline 96 WEYBURN & Dec. 18 & -5 & 2 & 0 & 10 & 0 & 1 & mostly clear & mostly clear \\
\hline 97 WHITE BEAR & Jan. 01 & -5 & -2 & 0 & 10 & 3 & 10 & partly & mostly clear \\
\hline 98 WHITEWOOD & Dec. 19 & -24 & -15 & 30 & 70 & 1 & 3 & mod. snow & light snow \\
\hline 99 YORKTON & Dec. 31 & -20 & -15 & 0 & 10 & 10 & 15 & overcast & \\
\hline
\end{tabular}




\begin{tabular}{|c|c|c|c|c|c|c|c|c|c|c|c|c|c|c|c|c|c|c|c|c|}
\hline \multirow[b]{2}{*}{$L O C . A L I T Y$} & \multicolumn{6}{|c|}{ EFFORT } & \multicolumn{14}{|c|}{ HABITAT } \\
\hline & 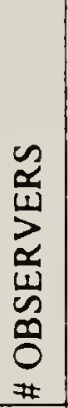 & $\mid \begin{array}{l}5 \\
8 \\
0 \\
z \\
0 \\
z\end{array}$ & $\mid \begin{array}{l}0 \\
8 \\
z \\
0 \\
2 \\
5 \\
5 \\
0 \\
=\end{array}$ & 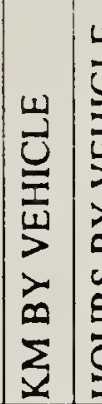 & 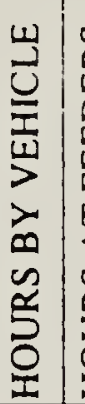 & 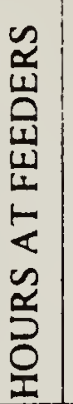 & 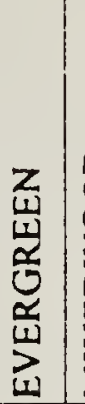 & 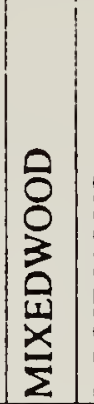 & 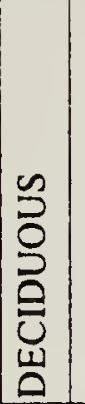 & 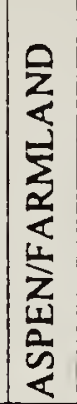 & 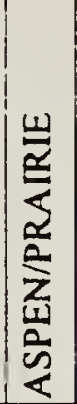 & 茪 & 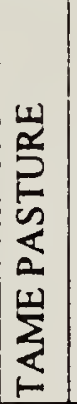 & 袋 & 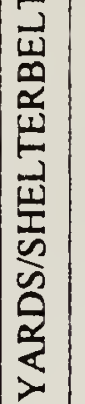 & $\mid \begin{array}{l}z \\
z \\
\frac{1}{2} \\
\frac{2}{5}\end{array}$ & 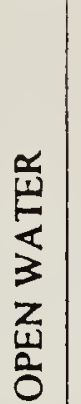 & $\frac{\mathrm{J}}{\mathrm{U}}$ & 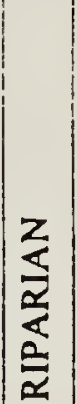 & $\mid \begin{array}{l}0 \\
y \\
y \\
z \\
z \\
z\end{array}$ \\
\hline ARCHERWILL & 5 & & & 70 & 2 & 5 & & 20 & & & & & & & 60 & 20 & & & & \\
\hline ARMIT & 5 & 3 & 3 & 160 & 6 & 1 & 10 & 40 & 40 & 10 & & & & & & & & & & \\
\hline ASSINIBOIA & 7 & & & & & 7 & & & & & & & & & 100 & & & & & \\
\hline AVONLEA & 1 & & & 96 & 3 & & & & & & & 40 & & & 5 & & & & & \\
\hline BANGOR & 8 & 3 & 2.5 & 95 & 7 & 7 & & & & 10 & 10 & & & & 50 & & & & & \\
\hline BEAUVAL & 2 & & 1.5 & 1102 & 4.5 & 0.5 & 30 & 30 & 10 & & & & & & 10 & 10 & & & & 10 \\
\hline BlGGAR & 5 & & 4.75 & 217 & 9 & 2 & & & & 11 & & & & & 19 & 20 & & & & \\
\hline BIG RIVER & 2 & 2 & 1.5 & & & 1.5 & 50 & 25 & & & & & 25 & & & & & & & \\
\hline BIRCH HILLS & 4 & 3.5 & 1.66 & 108 & 4 & 3 & & & 15 & 25 & & & & 40 & 5 & 15 & & & & \\
\hline BODMIN & 1 & 2 & 1.5 & & & & & 100 & & & & & & & & 4 & & & & \\
\hline BROADVIEW & 3 & 2 & 1 & 115 & 5 & & & & 10 & 15 & & & 10 & & 20 & 10 & 5 & & & \\
\hline BROMHEAD & 2 & & & 113 & 5 & & & & & & & & & \begin{tabular}{|l|}
90 \\
\end{tabular} & 5 & 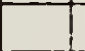 & & & 5 & \\
\hline CABRI & 1 & & & 1403. & 3.75 & 0.5 & & & & & & 5 & & & 10 & 5 & & & & \\
\hline CANDLE LAKE & 4 & 4 & & 200 & 9.5 & & & 70 & 20 & & & & & & & 10 & & & & \\
\hline CLARK'S CROSSING & 25 & 29 & 17.7 & 5773 & 31.3 & 10 & & 1 & 1 & 10 & 4 & 1 & 3 & & 31 & 19 & 4 & 1 & 15 & \\
\hline COCHIN & 3 & 4 & & 90 & 3 & & & & & 30 & 5 & & & 50 & 5 & 10 & & & & \\
\hline CORONACH & 1 & 4 & $3 / 2$ & 211 & 6 & & & & & & & 20 & & & 5 & 5 & 20 & & & \\
\hline CRAVEN & 14 & 10.5 & & 466 & 18 & 2 & & & 10 & 20 & & 5 & 10 & & 15 & 15 & 5 & & & \\
\hline CROOKED LAKE & 6 & 1 & 0.5 & 150 & 7 & 3 & & & 25 & 15 & & & & & 15 & & 40 & & & \\
\hline CROOKED RIVER & 2 & & & 25 & 1 & 7 & & & 33 & & 33 & & & & 34 & & & & & \\
\hline CYPRESS HILLS & 11 & 18 & 5 & 40 & 2 & 2 & 60 & 20 & & & 5 & 5 & & & 10 & & & & & \\
\hline DUCK LAKE & 2 & 8 & & $100:$ & 3.5 & & & 65 & 5 & 15 & & & & 5 & & 10 & & & & \\
\hline EASTEND & 5 & 3 & & 105 & 7 & 2 & & 10 & & & & 25 & & & 10 & 10 & & & & \\
\hline EMMA LAKE & 4 & 2 & 2 & 55 & 3 & 4 & & 95 & & & & & & & 5 & & & & & \\
\hline ESTEVAN & 3 & 5 & 2 & 160 & 8 & i & & & 25 & & & 10 & & 25 & 10 & 25 & 4 & 1 & & \\
\hline ESTUARY & 5 & 8 & & 100 & 5 & & & & & & 35 & 30 & 10 & 20 & \begin{tabular}{|l|}
5 \\
\end{tabular} & & & & & \\
\hline FENTON & 2 & 5 & 1.5 & 1556. & 6.25 & 1 & & & & 5 & & & & 75 & 15 & 5 & & & & \\
\hline FERLAND & 5 & & & 110 & 4 & & & & & & & 50 & 15 & 25 & 10 & & & & & \\
\hline FIFE LAKE & 2 & & & 100 & 4 & & & & & & & 10 & & & 15 & 2 & 50 & & & \\
\hline FORT QU'APPELLE & 20 & 1 & & 130 & 8 & 50 & & & & 20 & & & & & 20 & 20 & 20 & & 10 & \\
\hline FORT WALSH & & 331 & 16.5 & $\left.124\right|_{12} ^{2}$ & 12.8 & & & 56 & & & & 1 & & & 9 & & & & 26 & \\
\hline GARDINER DAM & 14 & 17 & 8.53 & 372 & 20 & & & & & 4 & & & & 40 & 35 & & 17 & & 4 & \\
\hline GOOD SPIRIT & 5 & 4 & 2 & 1047. & 7.751 & 1.25 & & 2 & 3 & 5 & 20 & 40 & 2 & & 20 & 2 & 1 & & & \\
\hline GOVENLOCK & 6 & 8 & & 216 & 13 & & & & & & & 50 & & 25 & 25 & & & & & \\
\hline GRASSLANDS & & 4 & & 96 & 8 & & & & & & & 50 & & 25 & 20 & 5 & & & & \\
\hline GRAYSON & 3 & 8 & & 70 & 5 & T & & & 5 & 35 & & & & 10 & 15 & 5 & 30 & & & \\
\hline HARRIS & 2 & 5 & 2.52 & 212 & 6 & & & & & 56 & & & & & 15 & 9 & & & & \\
\hline HEPBURN & 2 & & & 40 & 2 & 2 & & & 50 & & & & & & & 50 & & & & \\
\hline HOLBEIN & 2 & & & & & 5 & & & & & & & & 40 & 60 & & & & & \\
\hline INDIAN HEAD & 19 & 11 & & 140 & 8 & 72 & & & & 10 & 2 & & 5 & & 28 & 33 & 12 & & & \\
\hline KAMSACK & 23 & & & & & 20 & & 15 & 10 & & & & 25 & 40 & & 10 & & & & \\
\hline KELVINGTON & 3 & & & 55 & 2 & 7 & & & & 30 & & & & 60 & 10 & & & & & \\
\hline KENASTON & $T$ & 2 & & 30 & 4 & 1 & & & & 20 & & & 20 & 10 & 25 & 25 & & & & \\
\hline KENNEDY & 2 & 0.5 & 0.51 & 148 & 5 & 1 & & 5 & & & & & & 50 & 20 & 25 & & & & \\
\hline KENOSEE LAKE & 2 & 1 & & 176 & 5 & 1.5 & & 5 & 25 & 30 & 5 & & & & & 35 & & & & \\
\hline KILWINNING & 2 & $\mathrm{l}$ & & 168 & 6 & & 10 & 10 & & 30 & & & & 50 & & & & & & \\
\hline KINDERSLEY & 2 & 1 & 0.5 & 87 & 4 & & & & & & & & & & 30 & 60 & 2 & & & \\
\hline KINLOCH & 6 & 8 & 4 & 50 & 1 & 20 & 5 & 20 & 50 & 5 & & & & & 20 & & & & & \\
\hline KUTAWAGAN LAKE & 3 & 2 & & 260 & 8 & & & & & 20 & 10 & 10 & & & 10 & 10 & & & & \\
\hline LAIRD & 2 & 2 & & 55 & 4.5 & 2 & & 20 & & & & & & 40 & 20 & 20 & & & & \\
\hline
\end{tabular}


Table 2 - 2: COUNT COVERAGE

\begin{tabular}{|c|c|c|c|c|c|c|c|c|c|c|c|c|c|c|c|c|c|c|c|c|c|}
\hline \multirow[b]{2}{*}{$l, 0 x^{\prime} .4 J / I \gamma^{\prime}$} & \multicolumn{6}{|c|}{ EFFORT } & \multicolumn{15}{|c|}{ HABITAT } \\
\hline & 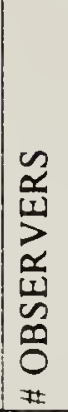 & $\left|\begin{array}{l}5 \\
0 \\
u \\
z \\
0 \\
z \\
z\end{array}\right|$ & $\mid \begin{array}{l}0 \\
0 \\
1 \\
z \\
0 \\
w \\
z \\
0 \\
0\end{array}$ & 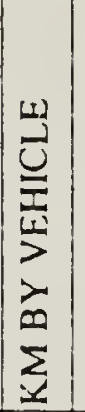 & 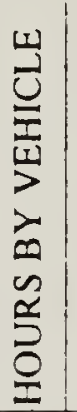 & 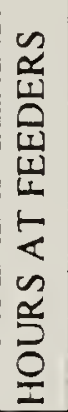 & 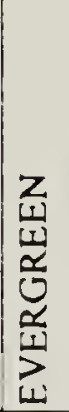 & 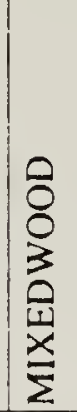 & 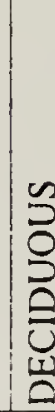 & $\frac{2}{z}$ & 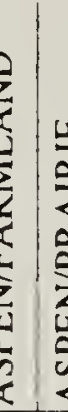 & $\begin{array}{l}\frac{y}{2} \\
\frac{2}{5} \\
\frac{2}{2} \\
\frac{1}{2} \\
\frac{1}{2}\end{array}$ & 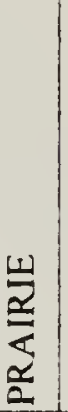 & 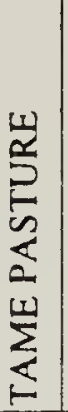 & 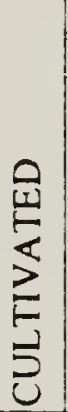 & 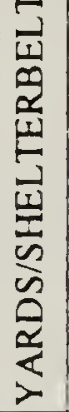 & $\mid \begin{array}{l}z \\
z \\
a \\
\underline{a}\end{array}$ & 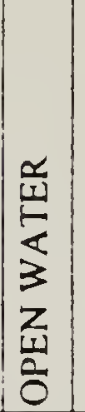 & 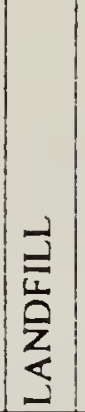 & 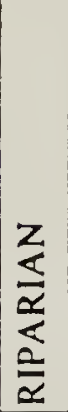 & $\begin{array}{l}0 \\
0 \\
\text { y } \\
x \\
w \\
ٍ \\
\Sigma\end{array}$ \\
\hline LAST MTN. LAKE & 4 & 3 & 2 & 160 & 7 & & & & & & 10 & & 30 & & 50 & 5 & 4 & 1 & & & \\
\hline LEADER $(\mathrm{N})$ & 1 & 3 & 3 & 20 & 3 & 1 & & & & & & 10 & 25 & 10 & 25 & 20 & & 10 & & & \\
\hline LEADER (S) & 2 & 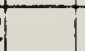 & & 100 & 5 & 2 & & & & & 40 & 10 & 10 & & & 20 & 10 & 10 & & & \\
\hline LIVELONG & 1 & & & & & 4 & & & & & & & & & & 100 & & & & & \\
\hline LOVE & 14 & & & 50 & 3 & 5 & & 25 & & & & & & & 25 & 25 & 25 & & & & \\
\hline LUSELAND & 6 & 14 & 6 & 250 & 5 & 4 & & & & & 20 & 10 & 5 & 5 & 15 & 20 & 25 & & & & \\
\hline MACDOWALL & 2 & & & 50 & 3 & 5 & & 20 & & & 50 & & & & & 30 & & & & & \\
\hline MATADOR & 2 & 6 & 2.5 & 80 & 6.5 & & & & & & & & 50 & 5 & 5 & & & & & 40 & \\
\hline MEADOW LAKE & 12 & 9 & 2 & 155 & 5 & 4 & & & 2 & 0 & 40 & & & & 10 & & 30 & & & & \\
\hline MELFORT & 1 & T & & & & & & & & & & & & & & & & & & & \\
\hline MELVILLE & 4 & & & 36 & 2 & 3 & & & & & 15 & & & & & 75 & 10 & & & & \\
\hline MISSINIPE & 1 & & & & 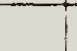 & 8 & & 100 & & & & & & & & & & & & & \\
\hline MOOSE JAW & & 8.5 & 9 & 117 & 5 & 8 & & & & & & & & & 10 & & 45 & & & 45 & \\
\hline MOOSE MOUNTAIN & 4 & 2 & & 141 & 4 & & & & 5 & 50 & 20 & 10 & & & & 10 & 10 & & & & \\
\hline NIPAWIN & 13 & & & 74 & 6 & $\overline{3}$ & 20 & 040 & & & 15 & 10 & & & & & 10 & 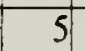 & & & \\
\hline NISBET FOREST & 5 & 2 & 1.5 & 64 & 3 & 4 & 10 & & & & & 85 & & & & 2.5 & 2.5 & & & & \\
\hline PIKE LAKE & & 34.5 & 23.3 & 4542 & 22.3 & 5 & & & & 51 & 10 & & 5 & & & 15 & 40 & & & & \\
\hline PORCUPINE PLAIN & $\overline{4}$ & 2 & 1 & 58 & 4 & $T$ & 10 & & & 0 & & & & & & 30 & 20 & & & & \\
\hline PREECEVILLE & 3 & t & & & & 3 & & & & & & & & & & 100 & & & & & \\
\hline PADDOCK WOOD & 5 & 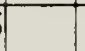 & & & & & & & & & & & & & & & & & & & \\
\hline PRINCE ALBERT & & $6.25 \mathrm{~F}$ & 4.25 & 2191 & 17.33 & 3.75 & & & & & 5 & 5 & & & & 5 & 85 & & & & \\
\hline PRINCE ALBERT NP & & 5 & 3 & 9 & 1.5 & & & & & & & & & & & & & & & & \\
\hline QU'APPELLE V. DAM & 3 & 2 & 1.9 & 225 & 5.6 & & & & & 01 & 10 & 10 & 5 & 15 & & 15 & 5 & 10 & & & \\
\hline RAYMORE & & 5 & 4 & 160 & 5 & 1 & & & & & 80 & & & & 10 & & 10 & & & & \\
\hline REGINA & & 48 & 21 & 657 & 31 & 4 & & & & 8 & 3 & 5 & 6 & & 30 & 22 & 26 & & & & \\
\hline ROCKGLEN & & 1 & 0.5 & 150 & 3 & & & & & & & & 10 & 40 & 40 & 10 & & & & & \\
\hline ROUND LAKE & & 1 & 3 & 242 & 8 & 1 & & & & & 40 & 10 & & & 25 & 5 & & 20 & & & \\
\hline SASK. LANDING PP & & 10 & 5 & 180 & 5 & & & & 1 & 0 & & & 30 & 10 & 35 & 10 & & 5 & & & \\
\hline SASK. RIVER FORKS & & 1 & 1 & 783 & 3.75 & & 10 & 10 & & 01 & 10 & & & 5 & 20 & 15 & & 10 & & & \\
\hline SASKATOON & 114 & 112 & & 8214 & 49.8 & 91 & 0.2 & & & 517 & .5 & 10 & 0.5 & 2 & & 16 & 48.8 & & 0.5 & 4 & \\
\hline SHAMROCK & & 1.5 & 1.5 & 175 & 7.5 & & & & & & & & & & & 30 & & & & & \\
\hline SKULL CREEK & 7 & & & 50 & 3 & 2 & & 10 & 0.1 & 0 & & 10 & 10 & 10 & & 10 & & & & & \\
\hline SNOWDEN & 23 & & & 181 & 6.5 & 6 & & 10 & & & & & & & & 40 & 20 & & & & \\
\hline SOMME & 9 & 2 & 1 & 138 & 6 & 25 & & 40 & & & & 30 & & & & 10 & 10 & & & & \\
\hline SPALDING & 5 & 5 & 3 & 80 & 5.5 & 3 & & 40 & & & & & & & & 20 & 40 & & & & \\
\hline SQUAW RAPIDS & 5 & 15 & & 130 & 10 & & 10 & 060 & & 9 & & & & & & 5 & & 10 & & & \\
\hline STOCKHOLM & 2 & 2 & 1 & 50 & 4 & 0 & & & & & 25 & 15 & & & & 20 & & & & & \\
\hline SWIFT CURRENT & & 43.5 & 21 & 3775 & 59.86 & 61.5 & & & & & & & 10 & & & 10 & 60 & & & & \\
\hline TISDALE & 3 & & & 20 & 1 & 2 & & & & & 25 & & & & & 25 & 25 & & & & \\
\hline TOGO & 3 & 1 & 1 & 5 & 1 & 8 & & & 8 & 01 & 10 & 10 & & & & & & & & & \\
\hline TORCH RIVER & 7 & & & 58 & 3 & 1 & & 25 & & & 25 & & & & & 25 & & & & & \\
\hline TURTLE LAKE & 2 & & & 50 & 2 & 10 & & 20 & & & & 20 & & & & 50 & & & & & \\
\hline WALDHEIM & 5 & & & 56 & 3 & 1 & & & & & & & & & & 15 & 15 & & & & \\
\hline WATSON & 4 & 1 & 1 & 20 & 1 & 7 & & & & & 20 & 20 & & & & 40 & 20 & & & & \\
\hline WELDON & 2 & & & & & 3 & & & & & & & & & & 100 & & & & & \\
\hline WEYBURN & 19 & 8 & 4 & 275 & 4 & 1 & & & & & & & 5 & & & 10 & 35 & & & & \\
\hline WHITE BEAR & 7 & 15 & 5 & 50 & 2 & 5 & & & & & & & 60 & & & 10 & & & & & \\
\hline WHITEWOOD & 18 & 3 & 2.5 & 3561 & 14.5 & 35 & & & & & 25 & & & & & 15 & 60 & & & & \\
\hline YORKTON & & 1 & 0.5 & 51 & 4 & & & & & & 20 & 20 & & & & 10 & 50 & & & & \\
\hline
\end{tabular}


Table 3-1. SPECIES RECORDED FROM NINE OR MORE LOCALITIES

$(0=$ a species recorded during count period but not seen on count day $)$

\begin{tabular}{|c|c|c|c|c|c|c|c|c|c|c|}
\hline 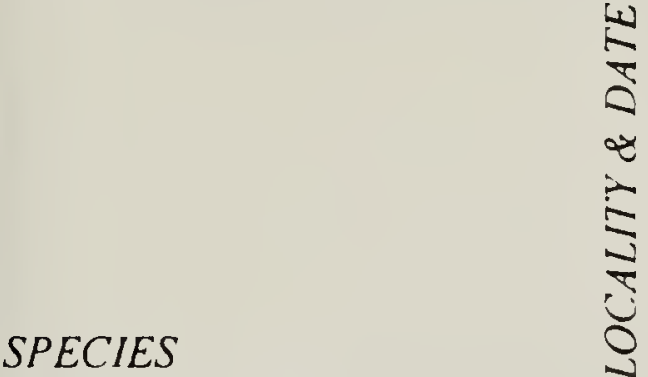 & 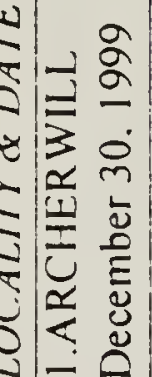 & 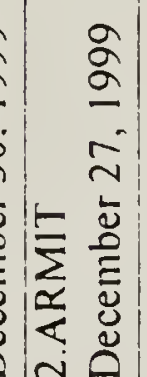 & 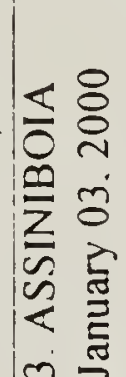 & 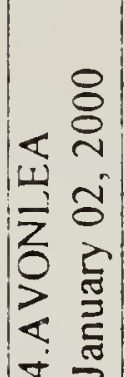 & 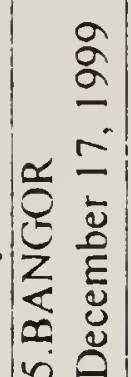 & 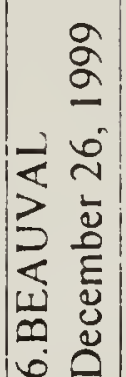 & 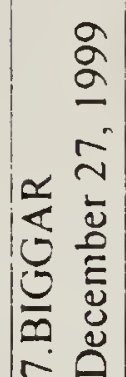 & & 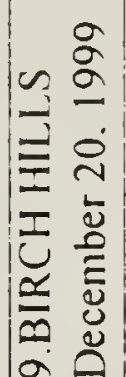 & 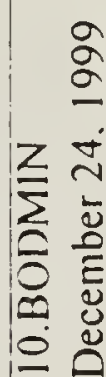 \\
\hline \multicolumn{11}{|l|}{ CANADA GOOSE } \\
\hline \multirow{2}{*}{\multicolumn{11}{|c|}{ COMMON GOLDENEYE }} \\
\hline & & & & & & & & & & \\
\hline BALD EAGLE & & 2 & 2 & & 2 & & 0 & & 0 & \\
\hline & & 1 & & & & & & & & \\
\hline \multirow{2}{*}{\multicolumn{11}{|c|}{ ROUGH-LEGGED HAWK }} \\
\hline & & & 4 & & & & & & & \\
\hline \multicolumn{11}{|l|}{$\begin{array}{l}\text { MERLIN } \\
\text { PRAIRIF FAICON }\end{array}$} \\
\hline \multicolumn{11}{|l|}{$\begin{array}{l}\text { PRAIRIE FALCON } \\
\text { GRAY PARTRIDGE }\end{array}$} \\
\hline \multicolumn{11}{|l|}{ RING-NECKED PHEASANT } \\
\hline \multirow{2}{*}{\multicolumn{11}{|c|}{$\begin{array}{l}\text { RUFFED GROUSE } \\
\text { SHARP-TAILED GROUSE }\end{array}$}} \\
\hline & & 5 & & & & & & & & \\
\hline \multicolumn{11}{|l|}{ ROCK DOVE } \\
\hline GREAT HORNED OWL & & 1] & 3 & & 0 & & 1 & & & \\
\hline \multirow{2}{*}{\multicolumn{11}{|c|}{$\begin{array}{l}\text { SNOWY OWL } \\
\text { SHORT-EARED OWL }\end{array}$}} \\
\hline & & & & & & & & & & \\
\hline \multirow{2}{*}{\multicolumn{11}{|c|}{$\begin{array}{l}\text { DOWNY WOODPECKER } \\
\text { HAIRY WOODPECKER }\end{array}$}} \\
\hline & & 1 & & & 8 & 2 & & & & \\
\hline \multicolumn{11}{|l|}{ PILEATED WOODPECKER } \\
\hline \multirow{5}{*}{$\begin{array}{l}\text { HORNED LARK } \\
\text { GRAY JAY } \\
\text { BLUE JAY } \\
\text { BLACK-BILLED MAGPIE } \\
\text { COMMON RAVFN }\end{array}$} & & & & 41 & & & 15 & & & \\
\hline & & 1 & i] & & & 11 & & 2 & & \\
\hline & 18 & 1 & & & & 6 & & 3 & & \\
\hline & 17 & 34 & 53 & 5 & 15 & 22 & 95 & & 47 & \\
\hline & & 58 & & & 13 & 66 & 18 & 13 & 12 & \\
\hline \multirow{4}{*}{$\begin{array}{l}\text { BLACK-CAPPED CHICKADEE } \\
\text { BOREAL CHICKADEE } \\
\text { RED-BREASTED NUTHATCH } \\
\text { WHITE-BREAST NUTHATCH }\end{array}$} & 42 & 29 & 2 & & 41 & 9 & 21 & 4 & 34 & \\
\hline & & 1 & & & & 6 & & & & \\
\hline & & 2 & & & 4 & 1 & 9 & 3 & & \\
\hline & & 3 & & & & & & & & \\
\hline \multirow{2}{*}{\multicolumn{11}{|c|}{\begin{tabular}{|l} 
GOLDEN-CROWNED KINGLET \\
AMERICAN ROBIN
\end{tabular}}} \\
\hline & & & & & & & 0 & & & \\
\hline \multicolumn{11}{|l|}{ BOHEMIAN WAXWING } \\
\hline \multirow{2}{*}{\multicolumn{11}{|c|}{$\begin{array}{l}\text { CEDAR WAXWING } \\
\text { NORTHERN SHRIKE }\end{array}$}} \\
\hline & & 1 & & & & & & & & \\
\hline \multicolumn{11}{|l|}{ EUROPEAN STARLING } \\
\hline \multirow{2}{*}{\multicolumn{11}{|c|}{$\begin{array}{l}\text { AMERICAN TREE SPARROW } \\
\text { DARK-EYED JUNCO (S-C) }\end{array}$}} \\
\hline & & & & & & & & & & \\
\hline \multicolumn{11}{|l|}{$\begin{array}{l}\text { SNOW BUNTING } \\
\text { PNOW }\end{array}$} \\
\hline PINE GROSBEAK & 54 & 25 & & & & 9 & & & & \\
\hline HOUSE FINCH & & & & & & & 18 & & & \\
\hline COMMON REDPOLL & 123 & 895 & & 116 & 226 & 127 & 282 & & 96 & 18 \\
\hline HOARY REDPOLL & & 11 & & & & 8 & 4 & & 2 & \\
\hline PINE SISK'IN & & & & & & 6 & & & & $c$ \\
\hline EVENING GROSBEAK & 73 & 25 & & & & 50 & & & & \\
\hline HOUSE SPARROW & 160 & & 205 & & 59 & & 332 & & 149 & \\
\hline INDIVIDUALS ON COUNT DAY & 926 & 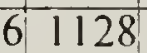 & 353 & 185 & 435 & 345 & 1094 & 28 & 578 & 32 \\
\hline SPECIES ON COUNT DAY & 21 & I. 19 & 9 & 7 & 14 & 20 & 17 & 8 & 19 & \\
\hline SPECIES COUNT PERIOD & 21 & 20 & 9 & 7 & 17 & 21 & 21 & 8 & 20 & IC \\
\hline INDIVIDUALS IN TABLES $4 \& 5$ & 1 & 0 & 0 & 0 & 4 & 17 & 51 & 0 & 2 & \\
\hline SPECIES IN TABLES $4 \& 5$ & & 1 & t) & 0 & 2 & 5 & 2 & 0 & & \\
\hline
\end{tabular}


Table 3-2. SPECIES RECORDED FROM NINE OR MORE LOCALITIES

$(0=$ a species recorded during count period but not seen on count day)

\begin{tabular}{|c|c|c|c|c|c|c|c|c|c|c|}
\hline \multirow[t]{14}{*}{ 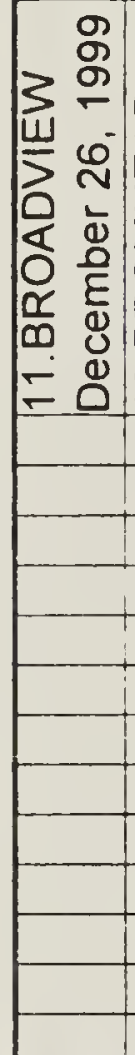 } & \multirow[t]{2}{*}{ 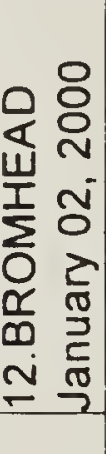 } & 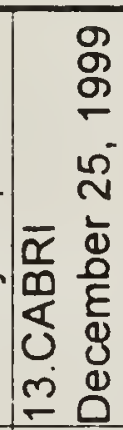 & \multicolumn{2}{|c|}{ 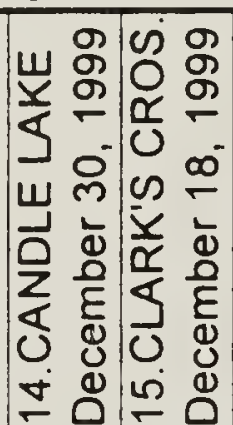 } & 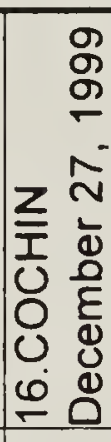 & \multicolumn{2}{|c|}{ 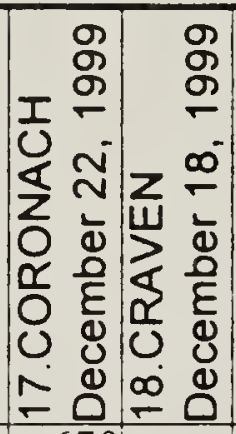 } & \multicolumn{2}{|c|}{ 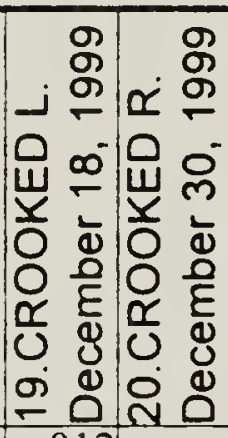 } & \multirow[t]{2}{*}{ 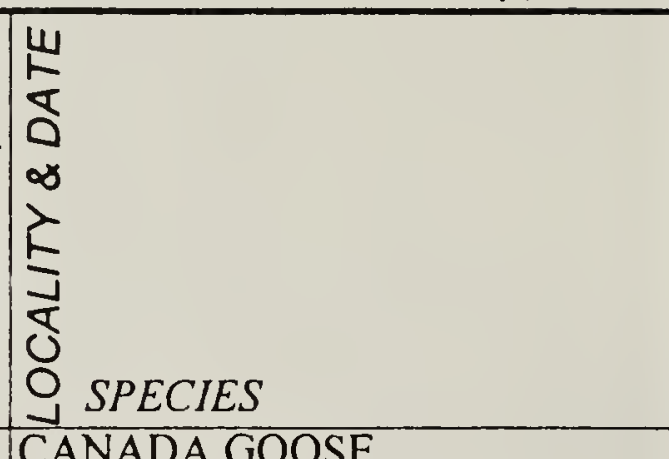 } \\
\hline & & & & 1105 & & 670 & & 812 & & \\
\hline & & & & 1 & & 7950 & 1] & 143 & & MALLARD \\
\hline & & & & 81 & & 30 & 3 & 4 & & COMMON GOLDENEYE \\
\hline & & & & 4 & & 1 & 3 & 3 & & BALD EAGLE \\
\hline & & & & 2 & & & $\overrightarrow{1}$ & $T$ & & NORTHERN GOSHAWK \\
\hline & & & & l & & 3 & 5 & & & ROUGH-LEGGED HAWK \\
\hline & 1] & & & & & 2 & 2 & l! & & GOLDEN EAGLE \\
\hline & & & & & & & & & & MERLIN \\
\hline & 2 & & & & & 1 & & & & PRAIRIE FALCON \\
\hline & & 5 & & 35 & 16 & 46 & 11] & & & GRAY PARTRIDGE \\
\hline & 12 & & & & & 5 & & & & RING-NECKED PHEASANT \\
\hline & & & & & 1 & & & 1 & & RUFFED GROUSE \\
\hline & 6 & 20 & & 19 & 6 & 35 & 2 & & & SHARP-TAILED GROUSE \\
\hline \multirow[t]{10}{*}{46} & 2 & 26 & & 204 & 40 & $\overline{53}$ & 76 & 80 & & ROCK DOVE \\
\hline & 4 & & & 2 & 3 & 2 & & 1 & & GREAT HORNED OWL \\
\hline & 1 & l] & & 2 & 1 & 2 & & & & SNOWY OWL \\
\hline & & & & & & 1 & & & & SHORT-EARED OWL \\
\hline & T & & 1 & 5 & 1 & l & 15 & 4 & & DOWNY WOODPECKER \\
\hline & & & & 13 & & & 7 & 4 & & HAIRY WOODPECKER \\
\hline & & & & 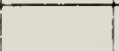 & 2 & & & & & PILEATED WOODPECKER \\
\hline & 82 & 31 & & & 25 & 363 & & & & HORNED LARK \\
\hline & & & 6 & & & & & & & GRAY JAY \\
\hline & & & 2 & 2 & 1 & & 14 & 10 & & BLUE JAY \\
\hline 24 & 4 & 25 & 7 & 311 & 12 & 141 & 101 & 42 & & BLACK-BILLED MAGPIE \\
\hline \multirow{3}{*}{$\begin{array}{r}13 \\
7\end{array}$} & & & 200 & 36 & 5 & & 2 & 38 & & COMMON RAVEN \\
\hline & & & 60 & 102 & 2 & 2 & 73 & 114 & & BLACK-CAPPED CHICKADEE \\
\hline & & & 22 & & & & & & & BOREAL CHICKADEE \\
\hline \multirow{4}{*}{$\begin{array}{l}2 \\
1\end{array}$} & & 0 & 2 & 7 & 2 & & 5 & 4 & & RED-BREASTED NUTHATCH \\
\hline & & & & & & & 10 & 2 & & WHITE-BREAST NUTHATCH \\
\hline & & & & & & & 3 & 2 & & GOLDEN-CROWNED KINGLET \\
\hline & & & & & & 1 & $\overline{0}$ & 10 & & AMERICAN ROBIN \\
\hline \multirow[t]{6}{*}{0} & & 10 & & 0 & 50 & 52 & & 368 & & BOHEMIAN WAXWING \\
\hline & & & & & & & t & 38 & & CEDAR WAXWING \\
\hline & & & & 1 & & 1 & 3 & & & NORTHERN SHRIKE \\
\hline & 4 & 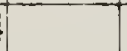 & & 33 & & 7 & 7 & 6 & & EUROPEAN STARLING \\
\hline & & & & ? & & 3 & & & & AMERICAN TREE SPARROW \\
\hline & & & & 1 & & 1 & 2 & & & DARK-EYED JUNCO (S-C) \\
\hline \multirow[t]{2}{*}{3} & 773 & & & 70 & 15 & 855 & 2 & & & SNOW BUNTING \\
\hline & & & 2 & & & & & & & PINE GROSBEAK \\
\hline \multirow{2}{*}{$\begin{array}{r}6 \\
161\end{array}$} & & & & & & & & & & HOUSE FINCH \\
\hline & 41 & 30 & 123 & 2100 & 7 & 387 & 245 & 143 & & COMMON REDPOLL \\
\hline 3 & 5 & & & 24 & & 2 & 15 & 2 & & HOARY REDPOLL \\
\hline & & & & & & & 3 & & & PINE SISKIN \\
\hline & & & 5 & & & & & & & EVENING GROSBEAK \\
\hline 112 & 90 & 129 & & 998 & 9 & 710 & 456 & 105 & & HOUSE SPARROW \\
\hline 379 & 1950 & 291 & 433 & 5160 & 198 & 11394 & 1079 & 1949 & 204 & INDIVIDUALS ON COUNT DAY \\
\hline 12 & 15 & 10 & 12 & 26 & 18 & 42 & 28 & 30 & & SPECIES ON COUNT DAY \\
\hline 13 & 15 & 11 & 12 & 28 & 18 & 42 & 30 & 30 & & SPECIES COUNT PERIOD \\
\hline 0 & 923 & 14 & 3 & l & 0 & 67 & 13 & 12 & & INDIVIDUALS IN TABLES $4 \& 5$ \\
\hline 0 & 2 & 1) & 1 & 2 & 0 & 14 & 4 & 6 & & SPECIES IN TABLES $4 \& 5$ \\
\hline
\end{tabular}


Table 3-3. SPECIES RECORDED FROM NINE OR MORE LOCALITIES

$(0=$ a species recorded during count period but not seen on count day $)$

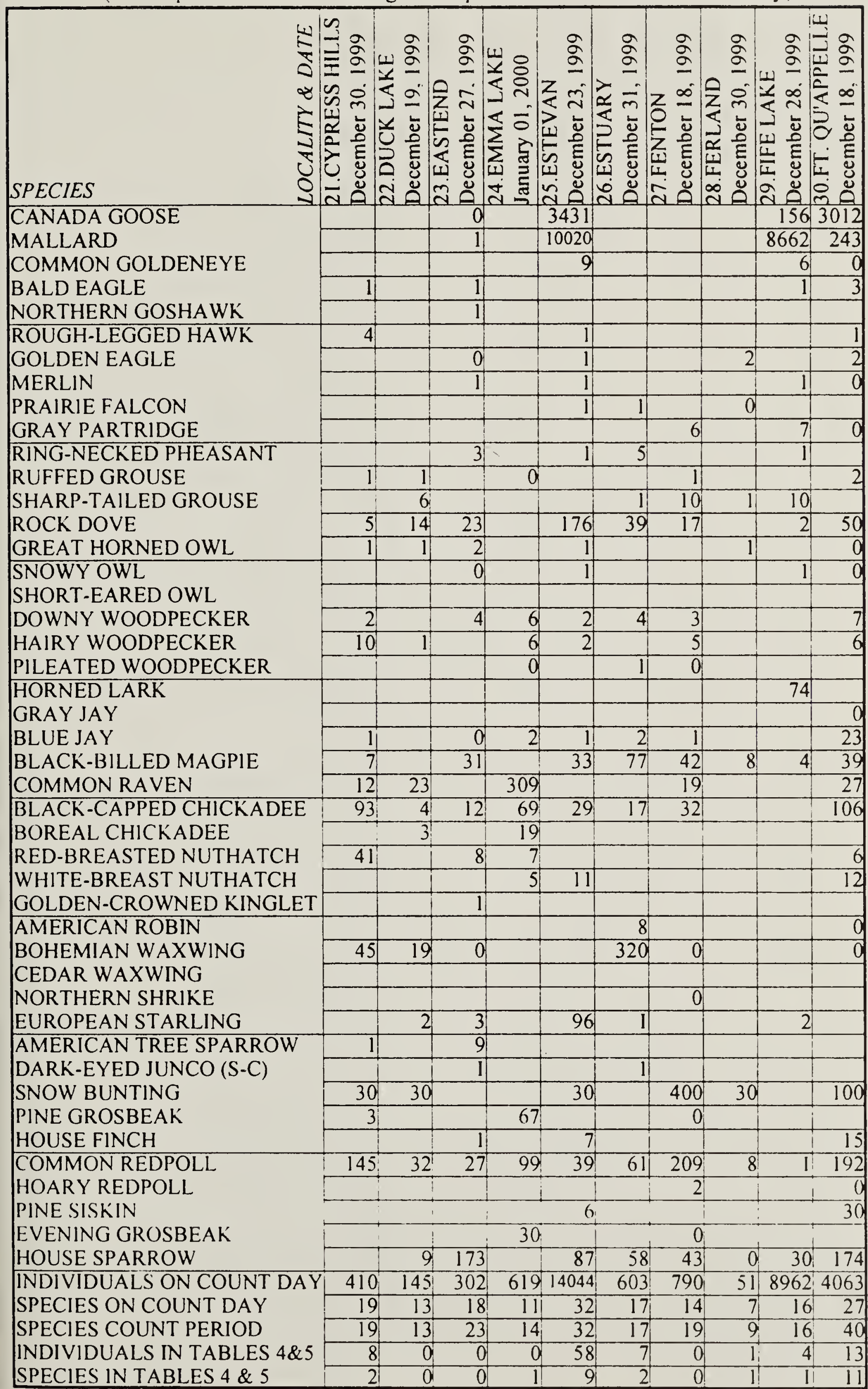


Table 3-4. SPECIES RECORDED FROM NINE OR MORE LOCALITIES

$(0=$ a species recorded during count period but not seen on count day)

\begin{tabular}{|c|c|c|c|c|c|c|c|c|c|c|}
\hline 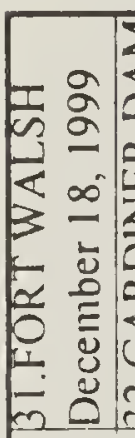 & 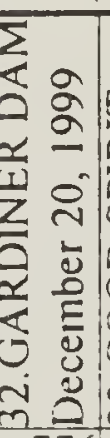 & 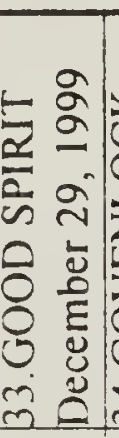 & 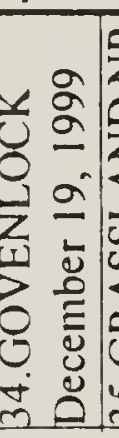 & 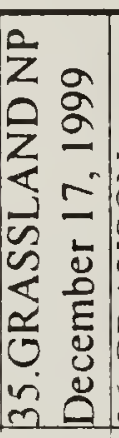 & 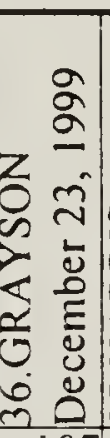 & 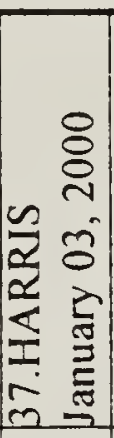 & 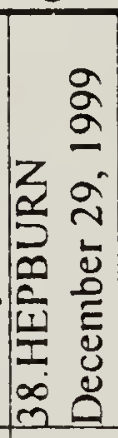 & 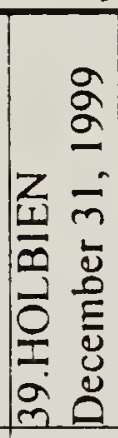 & 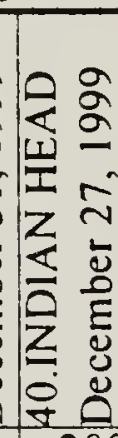 & : \\
\hline & 776 & & & & 107 & & & & & OCANADA GOOSE \\
\hline 13 & 7275 & & & & 157 & & & & & 7 MALLARD \\
\hline & 84 & & & & 3 & & & & & COMMON GOLDENEYE \\
\hline 5 & 33 & & I & & I & & & & & 5) BALD EAGLE \\
\hline 4 & & & & & & & & & & 1) NORTHERN GOSHAWK \\
\hline 2 & & & & & & & & & & ROUGH-LEGGED HAWK \\
\hline 5 & 2 & & & 1] & & 1 & & & & GOLDEN EAGLE \\
\hline 1 & & & & & & & & & & TMERLIN \\
\hline & & & 1 & & & & & & & TPRAIRIE FALCON \\
\hline 13 & 49 & & & 12 & & 17 & & & & GRAY PARTRIDGE \\
\hline 5 & & & 8 & 4 & & & & & & RING-NECKED PHEASANT \\
\hline 7 & & 4 & & & 1 & & & & & 2 RUFFED GROUSE \\
\hline 0 & 194 & & 8 & 33 & 0 & 172 & & & & 5 SHARP-TAILED GROUSE \\
\hline & 90 & 2 & & & 63 & 83 & & & & ROCK DOVE \\
\hline 5 & 3 & & 5 & 1] & & 2 & & & & 2 GREAT HORNED OWL \\
\hline & 3 & & 7 & & 1] & & & & & SNOWY OWL \\
\hline & & & & & & 5 & & & & SHORT-EARED OWL \\
\hline & 3 & ] & 1 & & 3 & 2 & 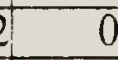 & & 2 & 9 DOWNY WOODPECKER \\
\hline 1 & 1 & 1 & & & 2 & & 0 & & 2 & 8 HAIRY WOODPECKER \\
\hline & & 1 & & & & & & & & PILEATED WOODPECKER \\
\hline 5 & I) & & 712 & 15 & & 8 & & & & HORNED LARK \\
\hline & & & & & & & & & & GRAY JAY \\
\hline & & 5 & & & 7 & & & & 4 & 2 BLUE JAY \\
\hline 197 & 249 & 42 & 26 & 46 & 23 & 183 & 3 & & 52 & BLACK-BILLED MAGPIE \\
\hline & 8 & 28 & & & 3 & 7 & 0 & & 11 & COMMON RAVEN \\
\hline 78 & 23 & 23 & & 1 & 20 & 20 & 3 & 11 & 60 & BLACK-CAPPED CHICKADEE \\
\hline & & & & & & & & & 1 & BOREAL CHICKADEE \\
\hline 12 & 6 & 2 & & & & & 0 & & 27 & 7 RED-BREASTED NUTHATCH \\
\hline & & $\mathrm{l}$ & & & & & & & 18 & 8) WHITE-BREAST NUTHATCH \\
\hline 15 & & & & & 10 & & & & & 1) GOLDEN-CROWNED KINGLET \\
\hline 0 & & 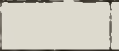 & & & 3 & & & & & AMERICAN ROBIN \\
\hline 129 & 30 & 12 & & & 60 & & & & & BOHEMIAN WAXWING \\
\hline 7 & & & & & & & & & & CEDAR WAXWING \\
\hline 2 & & & & ] & II & & & & I) & 1] NORTHERN SHRIKE \\
\hline 3 & ] & & 5 & 9 & & 7 & 7 & & & 1EUROPEAN STARLING \\
\hline 1 & 2 & & 3 & 20 & & & & & & AMERICAN TREE SPARROW \\
\hline & & & $\mathrm{I}$ & & & & 0 & & & DARK-EYED JUNCO (S-C) \\
\hline 78 & 320 & 48 & 47 & 3 & 22 & 4445 & & & & 5 SNOW BUNTING \\
\hline & & 5 & & & & & & & 8 & PINE GROSBEAK \\
\hline & & & & & & & & & & HOUSE FINCH \\
\hline 70 & 536 & 186 & 97 & 40 & 106 & 255 & 5 & 25 & 23 . & 5 COMMON REDPOLL \\
\hline & 2 & & & & & 1 & & & & 3 HOARY REDPOLL \\
\hline & & & & & & & & & & PINE SISKIN \\
\hline & & & & & & & & 35 & 5 & 2 EVENING GROSBEAK \\
\hline 185 & 889 & 20 & 263 & 48 & 47 & 242 & 0 & 0 & & 8HOUSE SPARROW \\
\hline 913 & 10895 & $38 \mathrm{I}$ & 1216 & 235 & 640 & 5450 & 0 & 94 & $4 \quad 122$ & 201 INDIVIDUALS ON COUNT DAY \\
\hline 36 & 34 & 16 & 21 & 15 & 20 & 16 & 6 & 13 & $3 \quad 31$ & 30 SPECIES ON COUNT DAY \\
\hline 38 & 34 & 16 & 21 & 15 & 23 & 16 & 6 & 13 & 31 & 30 SPECIES COUNT PERIOD \\
\hline 69 & 315 & 0 & 31 & 1 & 0 & 0 & 0 & 0 & 0 & 8 INDIVIDUALS IN TABLES $4 \& 5$ \\
\hline 13 & I1 & 0 & 7 & 1 & 2 & 0 & 0 & 0 & $\underline{0}$ & 4.SPECIES IN TABLES $4 \& 5$ \\
\hline
\end{tabular}


Table 3-5. SPECIES RECORDED FROM NINE OR MORE LOCALITIES

$(0=$ a species recorded during count period but not seen on count day $)$

SPECIES

CANADA GOOSE

MALLARD

COMMON GOLDENEYE

BALD EAGLE

NORTHERN GOSHAWK

ROUGH-LEGGED HAWK

GOLDEN EAGLE

MERLIN

PRAIRIE FALCON

GRAY PARTRIDGE

RING-NECKED PHEASANT

RUFFED GROUSE

SHARP-TAILED GROUSE

ROCK DOVE

GREAT HORNED OWL

SNOWY OWL

SHORT-EARED OWL

DOWNY WOODPECKER

HAIRY WOODPECKER

PILEATED WOODPECKER

HORNED LARK

GRAY JAY

BLUE JAY

BLACK-BILLED MAGPIE

COMMON RAVEN

BLACK'-CAPPED CHICKADEE

BOREAL CHICKADEE

RED-BREASTED NUTHATCH

WHITE-BREAST NUTHATCH

GOLDEN-CROWNED KINGLET

AMERICAN ROBIN

BOHEMIAN WAXWING

CEDAR WAXWING

NORTHERN SHRIKE

EUROPEAN STARLING

AMERICAN TREE SPARROW

DARK-EYED JUNCO (S-C)

SNOW BUNTING

PINE GROSBEAK

HOUSE FINCH

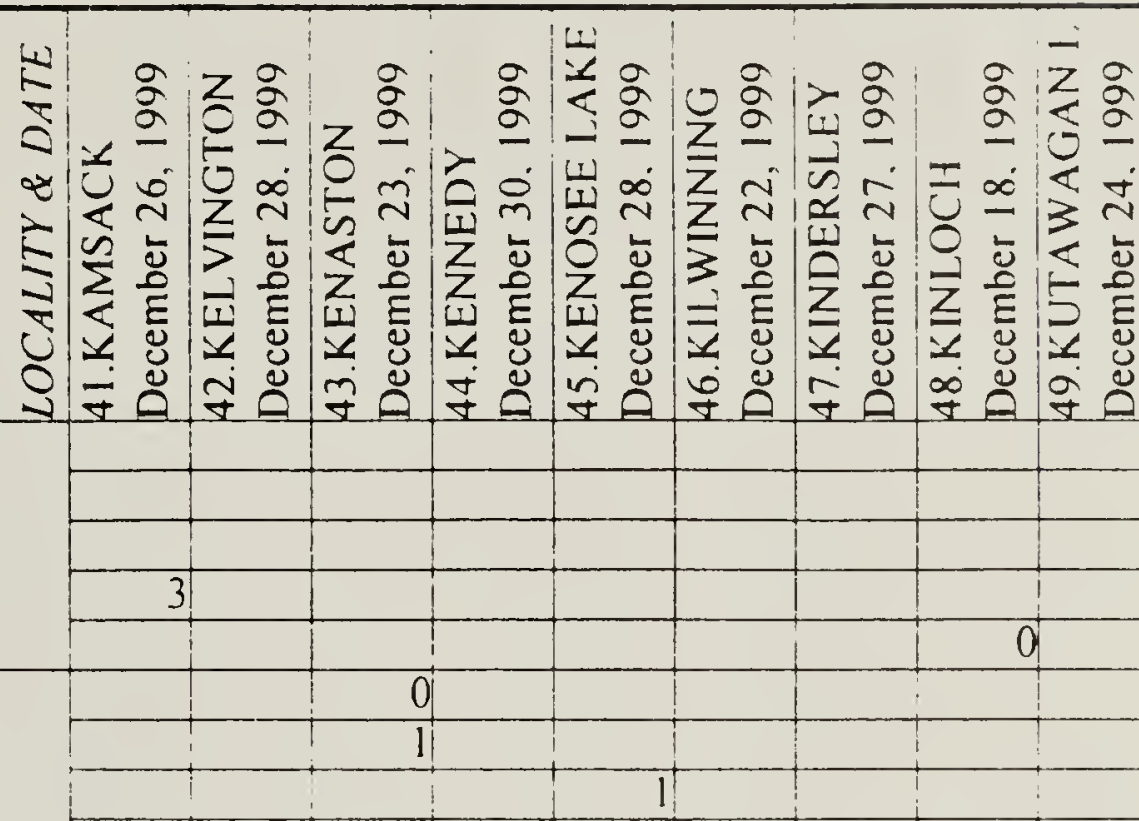

COMMON REDPOLL

HOARY REDPOLL

PINE SISK'IN

EVENING GROSBEAK'

HOUSE SPARROW

\begin{tabular}{l|l|l|l|l}
\hline & 16 & & 16 & 7 \\
\hline
\end{tabular}

\begin{tabular}{|r|r|r|r|r|r|r|r|r|}
\hline 8 & 4 & & & 1 & 4 & 5 & \\
\hline 12 & & 9 & & 11 & 7 & 0 & 7 \\
\hline & & 119 & 14 & 1 & 1 & 7 & 0 & 29 \\
\hline
\end{tabular}

INDIVIDUALS ON COUNT DAY

SPECIES ON COUNT DAY

SPECIES COUNT PERIOD

INDIVIDUALS IN TABLES 4 \&

SPECIES IN TABLES $4 \& 5$

\begin{tabular}{|r|r|r|r|r|r|r|r|r|}
\hline 2 & & 119 & 14 & 1 & 1 & 7 & 0 & 29 \\
\hline 2 & & 2 & & & & 1 & & 3 \\
\hline 25 & 1 & & 2 & 9 & 2 & & 4 & 1 \\
\hline 15 & 2 & 2 & 17 & 1 & & 5 & 1 \\
\hline
\end{tabular}

\begin{tabular}{|r|r|r|r|r|r|r|r|r|}
\hline & 0 & & & 1 & & & 2 & \\
\hline 8 & 2 & & & & & & & \\
\hline 38 & & & & 10 & 5 & & 8 & \\
\hline 16 & 7 & 22 & 22 & 32 & 58 & 33 & 9 & 110 \\
\hline 9 & 3 & 0 & & 7 & 29 & 15 & \\
\hline 87 & 6 & 4 & 29 & 64 & 24 & 37 & 4 \\
\hline 13
\end{tabular}

\begin{tabular}{|r|r|r|r|r|}
\hline 13 & & & 2 & 2 \\
\hline 22 & & 3 & 8 & \\
\hline 9 & & & 8 & 6 \\
\hline
\end{tabular}

\begin{tabular}{|r|r|r|r|r|r|r|r|r|}
\hline 4 & & & & & & & \\
\hline 100 & 60 & & & & & & 35 & 0 \\
\hline & & & & & & & & \\
\hline & & & & & & & & \\
\hline
\end{tabular}

\begin{tabular}{|c|c|c|c|c|c|c|}
\hline \multicolumn{7}{|c|}{132} \\
\hline 100 & 1000 & 82 & 10 & 200 & 35 & 105 \\
\hline 95 & & & & & 24 & \\
\hline
\end{tabular}

\begin{tabular}{|rrrrrrrrrr|}
\hline 563 & 60 & 125 & 132 & 209 & 155 & 5 & 145 & 133 \\
\hline 36 & & & 1 & & & & 26 & \\
\hline 68 & & & & & 1 & & & \\
\hline 308 & & & & 1 & 3 & & 104 & \\
\hline 145 & 3 & 65 & 406 & 89 & 141 & 50 & 27 & 425 \\
\hline 1820 & 1148 & 449 & 631 & 483 & 538 & 312 & 505 & 838 \\
\hline 25 & 11 & 12 & 12 & 21 & 18 & 7 & 18 & 15 \\
\hline 25 & 12 & 14 & 12 & 21 & 18 & 8 & 21 & 16 \\
\hline 0 & 0 & 1 & 11 & 6 & 0 & 0 & 0 & 0 \\
\hline 0 & 0 & 1 & 1 & 2 & 0 & 1 & 0 & 0 \\
\hline
\end{tabular}


Table 3-6. SPECIES RECORDED FROM NINE OR MORE LOCALITIES

$(0=$ a species recorded during count period but not seen on count day)

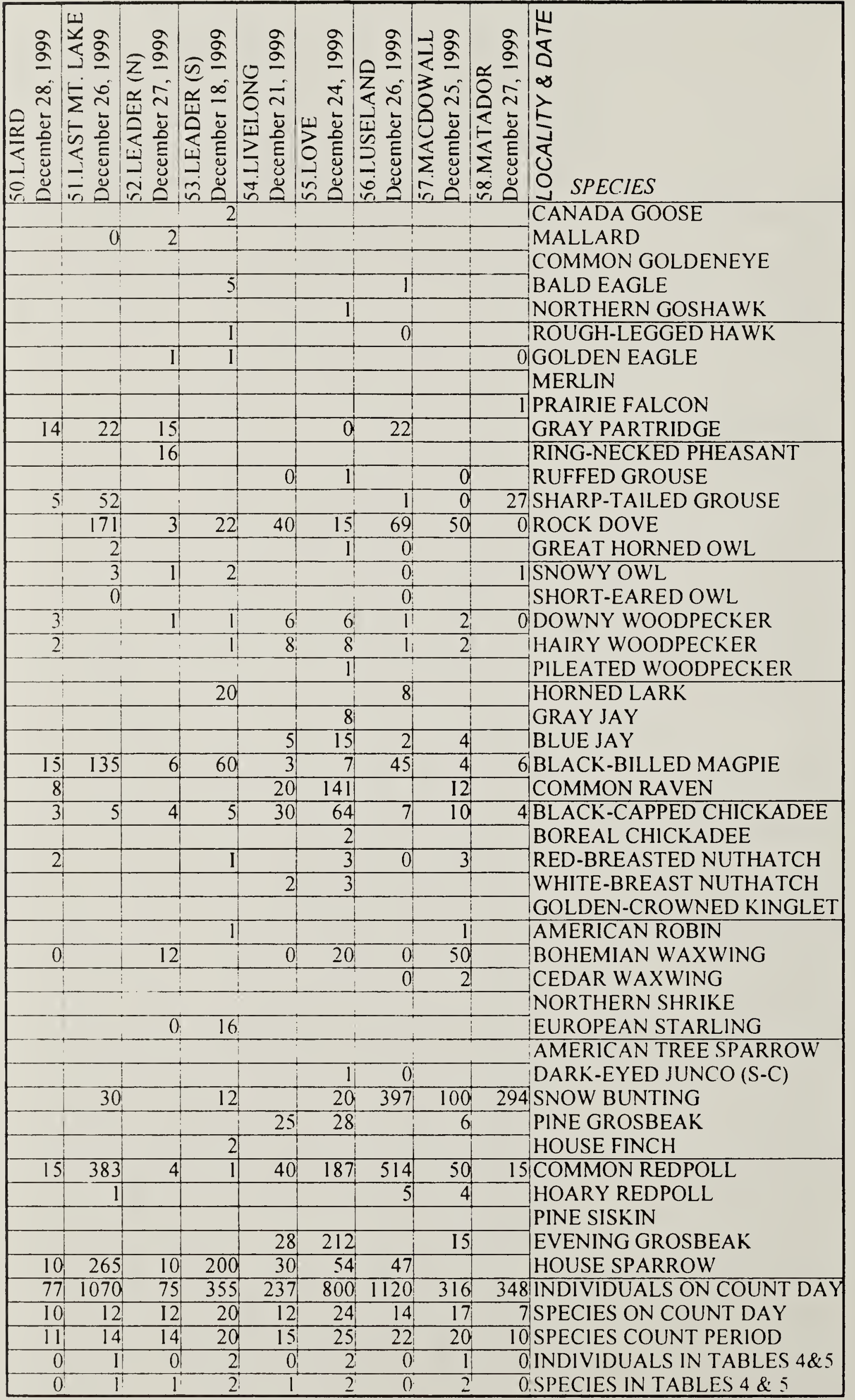


Table 3-7. SPECIES RECORDED FROM NINE OR MORE LOCALITIES

$(0=$ a species recorded during count period but not seen on count day $)$

SPECIES

CANADA GOOSE

MALLARD

COMMON GOLDENEYE

BALD EAGLE

NORTHERN GOSHAWK

ROUGH-LEGGED HAWK

GOLDEN EAGLE

MERLIN

PRAIRIE FALCON

GRAY PARTRIDGE

RING-NECKED PHEASANT

RUFFED GROUSE

SHARP-TAILED GROUSE

ROCK DOVE

GREAT HORNED OWL

SNOWY OWL

SHORT-EARED OWL

DOWNY WOODPECKER

HAIRY WOODPECKER

PILEATED WOODPECKER

HORNED LARK

GRAY JAY

BLUE JAY

BLACK-BILLED MAGPIE

COMMON RAVEN

BLACK-CAPPED CHICKADEE

BOREAL CHICKADEE

RED-BREASTED NUTHATCH

WHITE-BREAST NUTHATCH

GOLDEN-CROWNED KINGLET

AMERICAN ROBIN

BOHEMIAN WAXWING

CEDAR WAXWING

NORTHERN SHRIKE

EUROPEAN STARLING

AMERICAN TREE SPARROW

DARK-EYED JUNCO (S-C)

SNOW BUNTING

PINE GROSBEAK

HOUSE FINCH

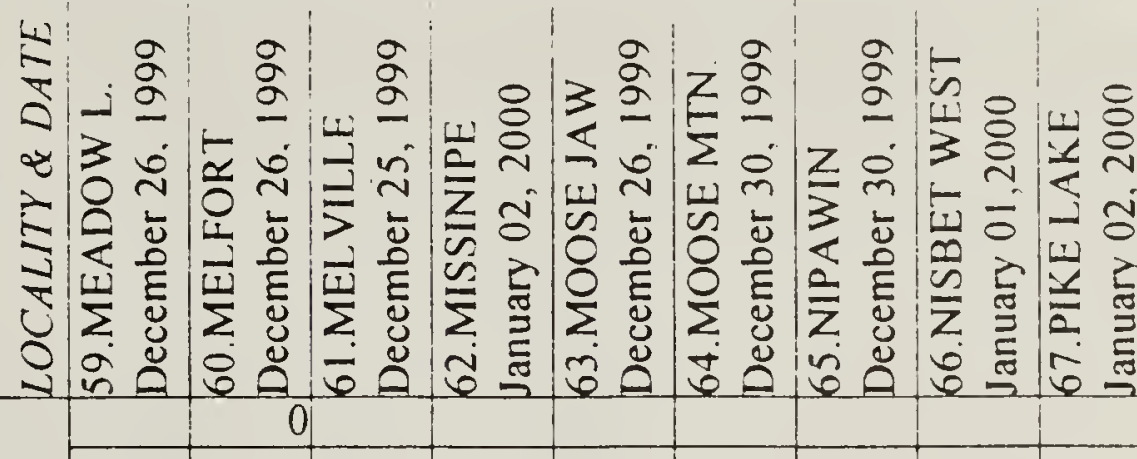

COMMON REDPOLL

HOARY REDPOLL

PINE SISKIN

EVENING GROSBEAK

HOUSE SPARROW

\begin{tabular}{|r|r|r|r|r|r|r|r|r|}
\hline & & & & & & & & \\
\hline & & & & & & & & \\
\hline & & & & & & & & \\
\hline & & & & 1 & 0 & & & \\
\hline
\end{tabular}

\begin{tabular}{|c|c|c|c|c|c|c|}
\hline & 7 & & & 6 & & \\
\hline & & 2 & & & & \\
\hline & & & & & 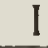 & \\
\hline 20 & & 20 & & & 0 & 48 \\
\hline 45 & 8 & 579 & 27 & 49 & & 48 \\
\hline
\end{tabular}

INDIVIDUALS ON COUNT DAY

SPECIES ON COUNT DAY

SPECIES COUNT PERIOD

INDIVIDUALS IN TABLES $4 \& 5$

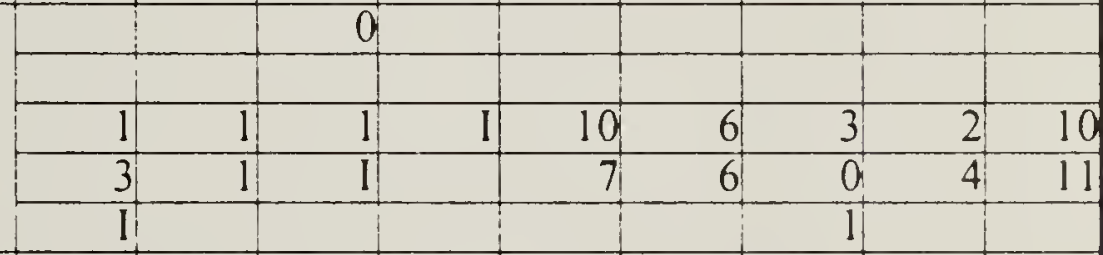

SPECIES IN TABLES 4 \& 5

\begin{tabular}{|c|c|c|c|c|c|c|c|c|}
\hline 3 & $i$ & & 2 & & & 1 & & \\
\hline 3 & & & 2 & I & 4 & 9 & 9 & 12 \\
\hline 15 & & 2 & & 62 & 33 & 5 & 3 & 14 \\
\hline 89 & 10 & 6 & 2 & & 4 & 151 & 8 & 4 \\
\hline 61 & 3 & 11 & 6 & 40 & 35 & 36 & 30 & 31 \\
\hline
\end{tabular}

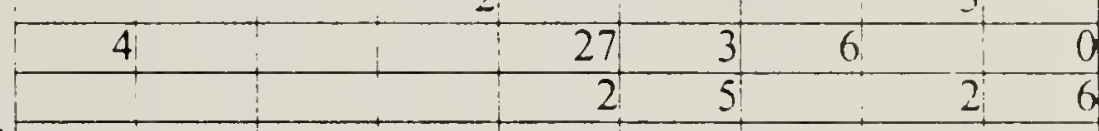

\begin{tabular}{|r|r|r|r|r|r|r|r|r|}
\hline & & & & & & & & 15 \\
\hline & 25 & 40 & & 0 & & 733 & 0 & 321 \\
\hline & & & & & & & & \\
\hline & & & & & & & 0 & 1 \\
\hline
\end{tabular}

\begin{tabular}{|c|c|c|c|c|c|c|c|c|}
\hline & & & & 20 & & & & \\
\hline & 25 & & & & & 75 & 350 & 288 \\
\hline 32 & 5 & & 12 & & & 48 & 16 & \\
\hline & & & & 27 & & & & \\
\hline $141^{\circ}$ & 12 & 30 & 27 & 31 & 383 & 360 & 216 & 1042 \\
\hline & & & & & & & 5 & 27 \\
\hline & & & & 2 & & & & \\
\hline 27 & 55 & & & & & 198 & 125 & \\
\hline & 50 & 40 & & 460 & 49 & 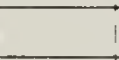 & & 302 \\
\hline 445 & 194 & 139 & 54 & 1299 & 598 & 1688 & 774 & 2639 \\
\hline 14 & 11 & 9 & 8 & 21 & 13 & 16 & 14 & 22 \\
\hline 14 & 12 & 10 & 8 & 24 & 13 & 17 & 18 & 23 \\
\hline 0 & 0 & 0 & 0 & 6 & 43 & 0 & 0 & \\
\hline 0 & 0 & 0 & 0 & 5 & 2 & 0 & 1 & \\
\hline
\end{tabular}


Table 3-8. SPECIES RECORDED FROM NINE OR MORE LOCALITIES

( $0=$ a species recorded during count period but not seen on count day)

\begin{tabular}{|c|c|c|c|c|c|c|c|c|}
\hline 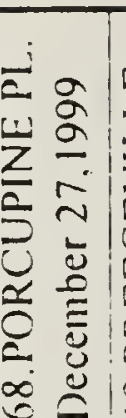 & 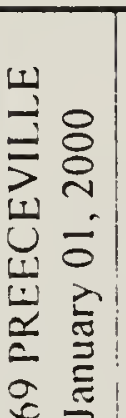 & 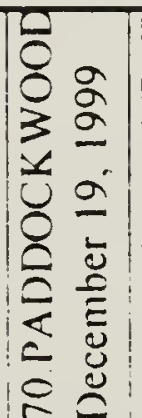 & 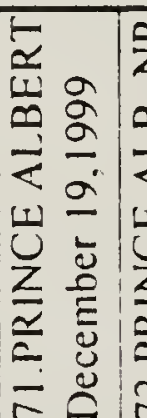 & 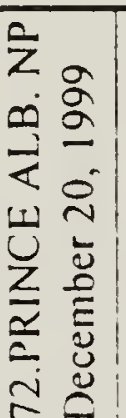 & 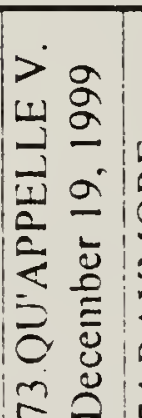 & 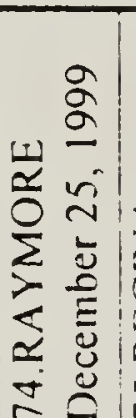 & 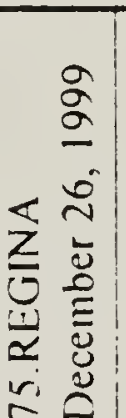 & 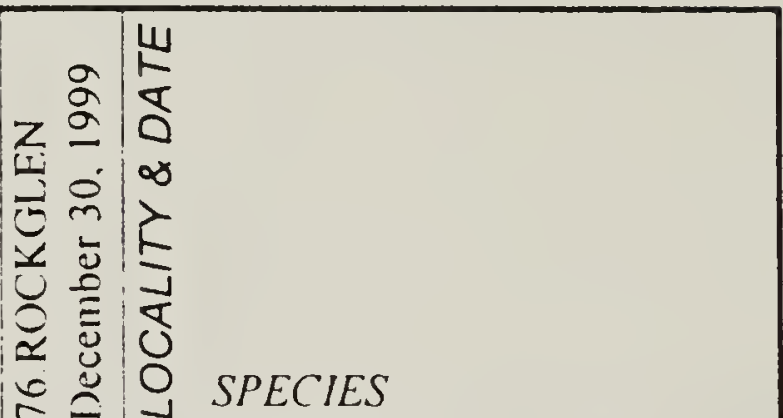 \\
\hline & & & & & 2220 & -0 & 2130 & $=0$ CANADA GOOSE \\
\hline & & & & & 192 & 0 & 4 & MALLARD \\
\hline & & & & & 3 & & & COMMON GOLDENEYE \\
\hline & & & 0 & & 3 & 0 & & BALD EAGLE \\
\hline & & & & & & 11 & & NORTHERN GOSHAWK \\
\hline & & & & & 7 & 0 & 0 & ROUGH-LEGGED HAWK \\
\hline & & & & & 1 & & & 0 GOLDEN EAGLE \\
\hline & & & 1 & & & 0 & 2 & MERLIN \\
\hline & & & & & & & 1 & PRAIRIE FALCON \\
\hline & & 11 & 6 & & & 4 & 54 & 26 GRAY PARTRIDGE \\
\hline & & & & & & & 6 & 0|RING-NECKED PHEASANT \\
\hline & & & & 2 & & 2 & & RUFFED GROUSE \\
\hline & & 2 & 7 & & & 2 & 1 & 19 SHARP-TAILED GROUSE \\
\hline & 14 & Ti: & 99 & & 18 & 59 & 764 & 2 ROCK DOVE \\
\hline & & 1 & 11 & & & 7 & 4 & GREAT HORNED OWL \\
\hline & & 2 & 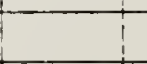 & & & 1 & 7 & SNOWY OWL \\
\hline & & & & & & T & & 0ISHORT-EARED OWL \\
\hline & 4 & 7 & 14 & & & it & 8 & DOWNY WOODPECKER \\
\hline & 4 & 9: & 13 & & & 3 & 2 & HAIRY WOODPECKER \\
\hline & 2 & 2 & 1 & & & & & PILEATED WOODPECKER \\
\hline & & & & & & 0 & I & 95 HORNED LARK \\
\hline & & 3 & 8 & 5 & & & & GRAY JAY \\
\hline & & 11 & 22 & & & & & BLUE JAY \\
\hline & 12 & 38 & 80 & & 27 & 73 & 208 & 3 BLACK-BILLED MAGPIE \\
\hline & 1) & & 126 & 4 & & 4 & & COMMON RAVEN \\
\hline & 8 & 22 & 165 & 25 & & 16 & 32 & BLACK-CAPPED CHICKADEE \\
\hline & & & 14 & 25 & & & & BOREAL CHICKADEE \\
\hline & & 3 & 8 & 2 & & i) & 38 & RED-BREASTED NUTHATCH \\
\hline I & & & 6 & & & & 8 & WHITE-BREAST NUTHATCH \\
\hline & & & & & & i) & 1 & GOLDEN-CROWNED KINGLET \\
\hline & & & 0 & & & i & 1) & AMERICAN ROBIN \\
\hline & 33 & & 1078 & & & 48 & 1: & BOHEMIAN WAXWING \\
\hline & & & & & & & & CEDAR WAXWING \\
\hline & & & 2 & & & 0 & & NORTHERN SHRIKE \\
\hline & & & & & & 42 & $\mid 91$ & EUROPEAN STARLING \\
\hline & & & & & & & & AMERICAN TREE SPARROW \\
\hline & & & 18 & & & 0 & 25 & DARK-EYED JUNCO (S-C) \\
\hline & & & 191 & & 378 & & 424 & SNOW BUNTING \\
\hline 4 & 6 & 47 & 47 & 11 & & 0 & & PINE GROSBEAK \\
\hline & & & & & & & 60 & HOUSE FINCH \\
\hline 36 & 117 & 214 & 555 & & 55 & 252 & 304 & 106 COMMON REDPOLL \\
\hline & & & & & & & 6 & HOARY REDPOLL \\
\hline & & & 31 & & & & 8 & PINE SISKIN \\
\hline 50 & 10 & 95 & 245 & & & & & EVENING GROSBEAK \\
\hline & 10 & 62 & 419 & & 60 & 541 & 1156 & HOUSE SPARROW \\
\hline 129 & 221 & 539 & 3159 & 102 & 2974 & 1067 & 5499 & 473 INDIVIDUALS ON COUNT DAY \\
\hline 10 & 12 & 17 & 27 & 11 & 12 & 21 & 35 & 9 SPECIES ON COUNT DAY \\
\hline 10) & 12 & 17 & 29 & $11 !$ & 12 & 34 & 36 & 15 SPECIES COUNT PERIOD \\
\hline 0 & 0 & 1 & 1. & 22 & 2 & 0 & 52 & 2201 INDIVIDUALS IN TABLES $4 \& 5$ \\
\hline 0 & 0 & & & & & & & 2 SPECIES IN TABLES $4 \& 5$ \\
\hline
\end{tabular}


Table 3-9. SPECIES RECORDED FROM NINE OR MORE LOCALITIES

$(0=$ a species recorded during count period but not seen on count day)

SPECIES

CANADA GOOSE

MALLARD

COMMON GOLDENEYE

BALD EAGLE

NORTHERN GOSHAWK

ROUGH-LEGGED HAWK

GOLDEN EAGLE

MERLIN

PRAIRIE FALCON

GRAY PARTRIDGE

RING-NECKED PHEASANT

RUFFED GROUSE

SHARP-TAILED GROUSE

ROCK DOVE

GREAT HORNED OWL

SNOWY OWL

SHORT-EARED OWL

DOWNY WOODPECKER

HAIRY WOODPECKER

PILEATED WOODPECKER HORNED LARK

GRAY JAY

BLUE JAY

BLACK-BILLED MAGPIE

COMMON RAVEN

BLACK-CAPPED CHICKADEE

BOREAL CHICKADEE

RED-BREASTED NUTHATCH

WHITE-BREAST NUTHATCH

GOLDEN-CROWNED KINGLET

AMERICAN ROBIN

BOHEMIAN WAXWING

CEDAR WAXWING

NORTHERN SHRIKE

EUROPEAN STARLING

AMERICAN TREE SPARROW

DARK-EYED JUNCO (S-C)

SNOW BUNTING

PINE GROSBEAK

HOUSE FINCH

COMMON REDPOLL

HOARY REDPOLL

PINE SISKIN

EVENING GROSBEAK

HOUSE SPARROW

INDIVIDUALS ON COUNT DAY

SPECIES ON COUNT DAY

SPECIES COUNT PERIOD

INDIVIDUALS IN TABLES 4\&5

SPECIES IN TABLES $4 \& 5$

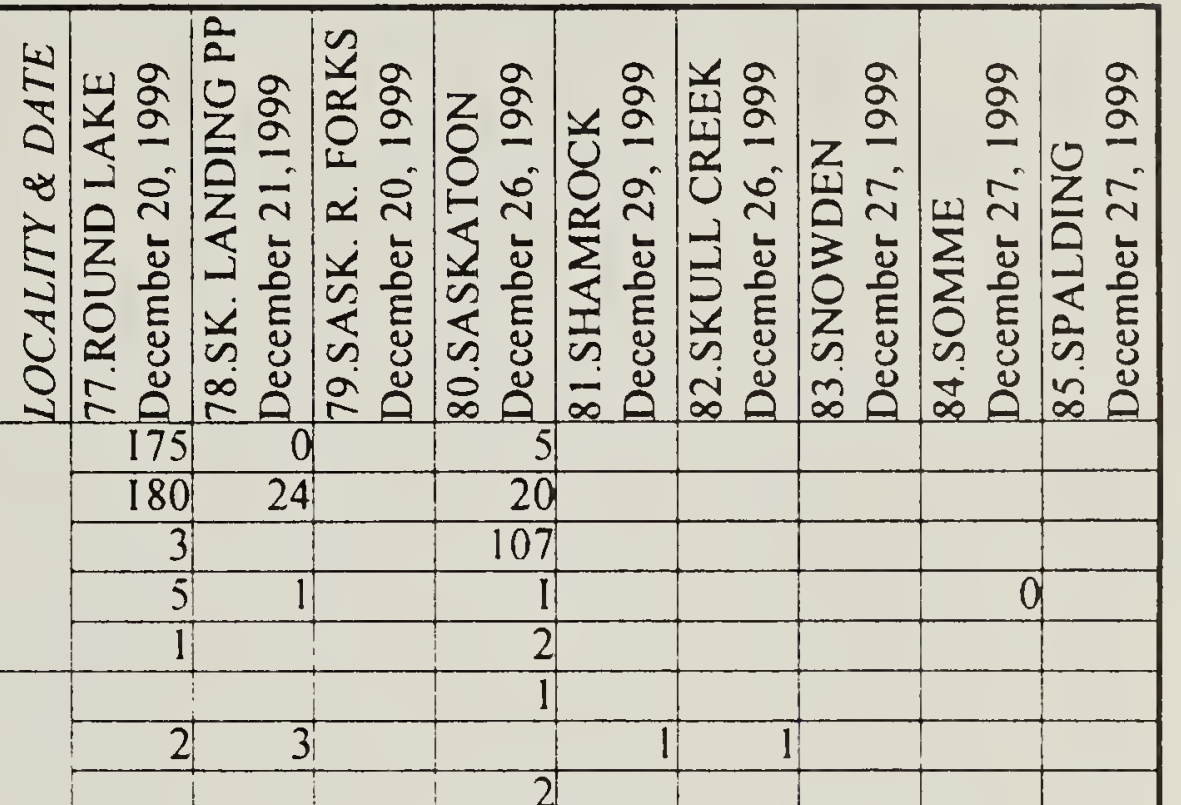

\begin{tabular}{|r|r|r|r|r|r|r|r|r|}
\hline & & & 2 & & & & \\
\hline & & & & & 1 & & & \\
\hline & 55 & 25 & 21 & 5 & 11 & 8 & \\
\hline 1 & 2 & & & & & & \\
\hline 5 & 111 & 104 & 2133 & 4 & 6 & 10 & 27 & 6 \\
\hline 2 & 2 & 3 & 1 & 2 & & 2 & \\
\hline & & & 0 & 1 & & & 0 \\
\hline
\end{tabular}

\begin{tabular}{|r|r|r|r|r|r|r|r|}
\hline $\mathrm{I}$ & & & & & & & \\
\hline 13 & $\mathrm{I}$ & & $3 \mathrm{I}$ & 4 & 10 & 2 & $\mathrm{I}$ \\
\hline 10 & & 3 & 26 & 1 & 19 & 3 & $\mathrm{I}$ \\
\hline & & 2 & & & 1 & 1 & \\
\hline
\end{tabular}

\begin{tabular}{|r|r|r|r|r|r|r|r|r|}
\hline 0 & 6 & & & 15 & 30 & & & \\
\hline & & & & & & 14 & 9 & \\
\hline 7 & & 2 & 64 & & 4 & 31 & 6 & \\
\hline 38 & 67 & 23 & 947 & 19 & 40 & 40 & 23 & 14 \\
\hline 13 & & 50 & 35 & & & 252 & 33 & 5 \\
\hline 97 & 12 & 17 & 632 & & 20 & 103 & 44 & 4 \\
\hline & & 1 & & & & 11 & \\
\hline
\end{tabular}

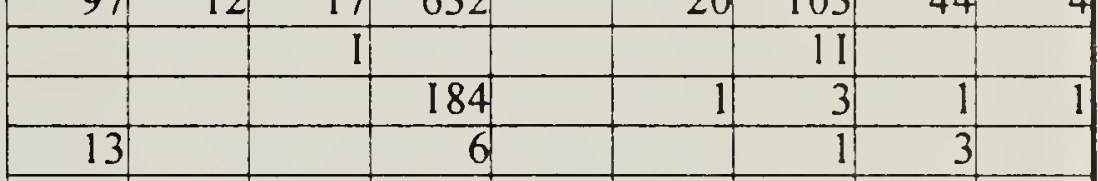

\begin{tabular}{|r|r|r|r|r|r|r|r|r|}
\hline 8 & 45 & 2 & 172 & & & 25 & 0 & \\
\hline 1 & & & 105 & & & & & \\
\hline 1 & & & 23 & & & & 4 & \\
\hline & & & 12 & & 30 & & & \\
\hline & 3759 & 20 & 6 & 60 & & 21 & 50 & \\
\hline 1 & & & 6 & & & 67 & 4 & \\
\hline 587 & 84 & 222 & 2252 & & & & & \\
\hline 3 & & & 16 & & & & 2 & 2 \\
\hline
\end{tabular}

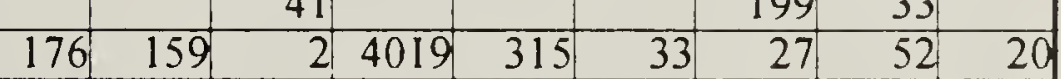

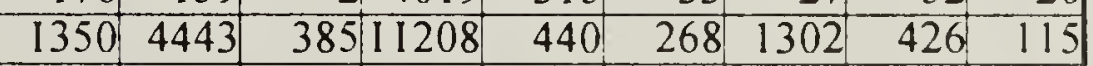

\begin{tabular}{|r|r|r|r|r|r|r|r|r|}
\hline 29 & 21 & 12 & 40 & $1 \mathrm{I}$ & 18 & 23 & 22 & 12 \\
\hline $3 \mathrm{I}$ & 22 & 12 & 44 & 11 & 18 & 25 & 25 & 14 \\
\hline 5 & 5 & 0 & 60 & 3 & 1 & 2 & 0 & 0 \\
\hline 5 & 4 & 0 & 14 & 2 & 1 & 4 & 0 & 1 \\
\hline
\end{tabular}


Table 3-10. SPECIES RECORDED FROM NINE OR MORE LOCALITIES

$(0=$ a species recorded during count period but not seen on count day)

\begin{tabular}{|c|c|c|c|c|c|c|c|c|c|}
\hline 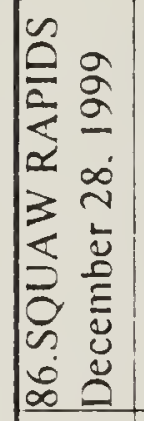 & 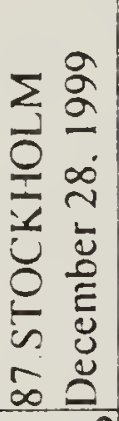 & 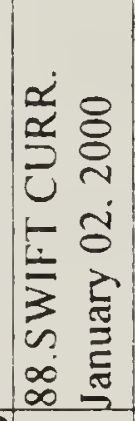 & 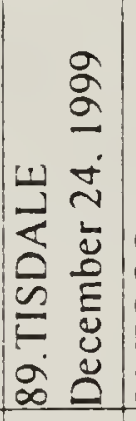 & 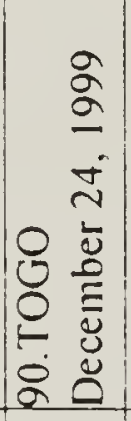 & 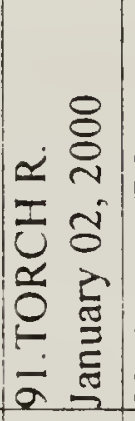 & 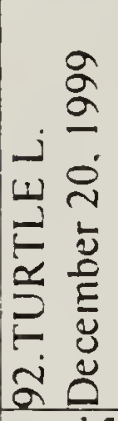 & 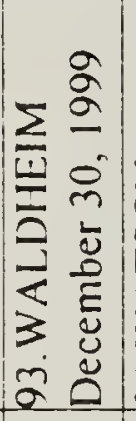 & 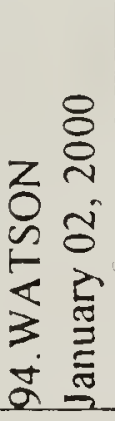 & $\begin{array}{l}w \\
w \\
\sigma \\
0 \\
\infty \\
z \\
\vdots \\
\vdots \\
\vdots \\
0 \\
0\end{array}$ \\
\hline & 2 & & & & & 15 & & & CANADA GOOSE \\
\hline 2 & & 1 & & & & & & & MALLARD \\
\hline 448 & & 1 & & & & & & & COMMON GOLDENEYE \\
\hline 6 & 2 & 0 & & & & & & & BALD EAGLE \\
\hline & & & & & & & & & NORTHERN GOSHAWK \\
\hline & & l & & & 0 & & & & ROUGH-LEGGED HAWK \\
\hline & & & & & & & & & GOLDEN EAGLE \\
\hline & & 0 & & & & & & & MERLIN \\
\hline & & 0 & & & & & & & PRAIRIE FALCON \\
\hline 3 & 0 & 123 & & & 8 & 7 & & & GRAY PARTRIDGE \\
\hline & & 1 & & & & & & & RING-NECKED PHEASANT \\
\hline 5 & & & & 1 & 10 & 15 & & & RUFFED GROUSE \\
\hline I1] & 3 & 11 & & & 10 & 25 & 12 & & 2 SHARP-TAILED GROUSE \\
\hline & 5 & 287 & & & & & II & & ROCK DOVE \\
\hline 2 & & 1 & & & & & & & GREAT HORNED OWL \\
\hline & 0 & 3 & & 1 & & 2 & 1 & & (0) SNOWY OWL \\
\hline & 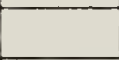 & 1 & & & & & & & SHORT-EARED OWL \\
\hline 3 & & 1 & 1 & 2 & 3 & 4 & 2 & & 1 DOWNY WOODPECKER \\
\hline 4 & 1 & & & 2 & 5 & 6 & 2 & & 2 HAIRY WOODPECKER \\
\hline 7 & & & & & 2 & 3 & & & PILEATED WOODPECKER \\
\hline & & 66 & & & & & & & HORNED LARK \\
\hline 36 & & & & & 5 & 7 & & & GRAY JAY \\
\hline 25 & & & & 2 & 8 & 15 & 4 & & BLUE JAY \\
\hline 63 & 4 & 118 & 2 & & 15 & 20 & 15 & & 3 BLACK-BILLED MAGPIE \\
\hline 89 & 10 & & 3 & & 39 & 40 & 9 & & COMMON RAVEN \\
\hline 28 & 25 & 0 & 2 & 10 & 30 & 7 & 7 & & 8BLACK-CAPPED CHICKADEE \\
\hline 18 & & & & & & 3 & & & BOREAL CHICKADEE \\
\hline 1 & & 9 & & & & 2 & 8 & & RED-BREASTED NUTHATCH \\
\hline 1 & & $\overrightarrow{0}$ & & & 4 & 4 & + & & WHITE-BREAST NUTHATCH \\
\hline & & 0 & & & & & & & GOLDEN-CROWNED KINGLET \\
\hline & & 0 & & & & & & & AMERICAN ROBIN \\
\hline 1 & & & 10 & 20 & & & 5 & & BOHEMIAN WAXWING \\
\hline & & 0 & & & & 50 & & & CEDAR WAXWING \\
\hline & & & & $\mathrm{I}$ & & & $\overline{0}$ & & NORTHERN SHRIKE \\
\hline & & & & & & & & & EUROPEAN STARLING \\
\hline & & I & & & & & & & AMERICAN TREE SPARROW \\
\hline & & 11 & & & & & & & DARK-EYED JUNCO (S-C) \\
\hline 50 & 500 & 1171 & & & 142 & 50 & 20 & & 7 SNOW BUNTING \\
\hline 44 & & & & & 19 & & & & PINE GROSBEAK \\
\hline & & 32 & & & & & 5 & & HOUSE FINCH \\
\hline 187 & & 23 & 8 & 30 & 43 & 100 & 75 & & 4COMMON REDPOLL \\
\hline 5 & $\mathrm{l}$ & & & & 6 & 35 & & & HOARY REDPOLL \\
\hline & & 5 & & & & 10 & & & PINE SISKIN \\
\hline 104 & & & & & 140 & 75 & & & EVENING GROSBEAK \\
\hline 25 & 25 & 2047 & 6 & & & & 50 & & 1 HOUSE SPARROW \\
\hline 1282 & 578 & 3932 & 32 & 69 & 489 & 501 & 226 & & 5 INDIVIDUALS ON COUNT DAY \\
\hline 34 & 11 & 22 & 7 & 9 & 17 & 25 & 15 & & 0 SPECIES ON COUNT DAY \\
\hline 34 & 14 & 32 & 7 & 9] & 19 & 25 & 17 & & ITSPECIES COUNT PERIOD \\
\hline 114 & 0 & 18 & 0 & 0 & 0 & 6 & 0 & & 7 INDIVIDUALS IN TABLES $4 \& 5$ \\
\hline 12 & 1 & 3 & 0 & 0 & 1 & 3 & 1 & & 2 SPECIES IN TABLES $4 \& 5$ \\
\hline
\end{tabular}


Table 3-11. SPECIES RECORDED FROM NINE OR MORE LOCALITIES

$(0=$ a species recorded during count period but not seen on count day')

\section{SPECIES}

CANADA GOOSE

MALLARD

COMMON GOLDENEYE

BALD EAGLE

NORTHERN GOSHAWK

ROUGH-LEGGED HAWK

GOLDEN EAGLE

MERLIN

PRAIRIE FALCON

GRAY PARTRIDGE

RING-NECKED PHEASANT

RUFFED GROUSE

SHARP-TAILED GROUSE

ROCK DOVE

GREAT HORNED OWL

SNOWY OWL

SHORT-EARED OWL

DOWNY WOODPECKER

HAIRY WOODPECKER

PILEATED WOODPECKER

HORNED LARK

GRAY JAY

BLUE JAY

BLACK-BILLED MAGPIE

COMMON RAVEN

BLACK-CAPPED CHICKADEE

BOREAL CHICKADEE

RED-BREASTED NUTHATCH

WHITE-BREAST NUTHATCH

GOLDEN-CROWNED KINGLET

AMERICAN ROBIN

BOHEMIAN WAXWING

CEDAR WAXWING

NORTHERN SHRIKE

EUROPEAN STARLING

AMERICAN TREE SPARROW

DARK-EYED JUNCO (S-C)

SNOW BUNTING

PINE GROSBEAK

HOUSE FINCH

COMMON REDPOLL

HOARY REDPOLL

PINE SISKIN

EVENING GROSBEAK

HOUSE SPARROW

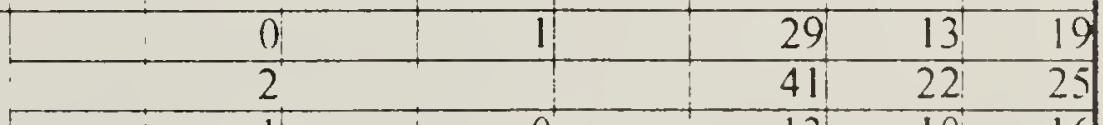

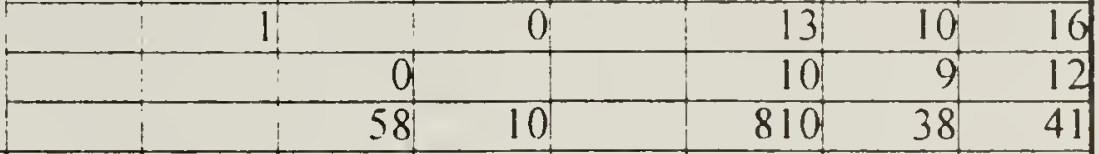

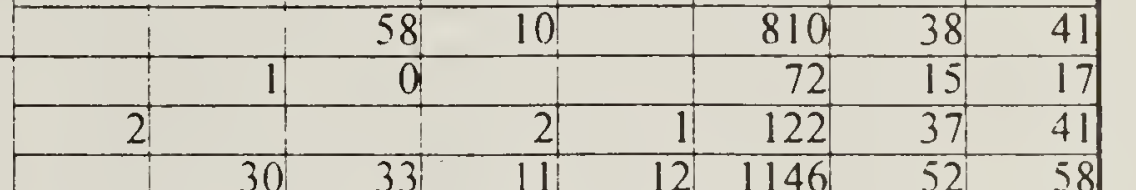

\begin{tabular}{|r|rrr|r|r|r|r|}
\hline & 30 & 33 & 11 & 12 & 1146 & 52 & 58 \\
\hline & 115 & 107 & 40 & 147 & 6746 & 66 & 68 \\
\hline 11 & 0 & 2 & 1 & 86 & 44 & 48 \\
\hline
\end{tabular}

\begin{tabular}{|r|r|r|r|r|r|r|r|}
\hline & 1 & 1 & & 1 & 60 & 31 & 40 \\
\hline 2 & & & 0 & & 17 & 5 & 9 \\
\hline 2 & 2 & 1 & 9 & 3 & 328 & 75 & 78 \\
\hline
\end{tabular}

\begin{tabular}{|c|c|c|c|c|}
\hline 29 & 1 & 1646 & 23 & 25 \\
\hline ? & 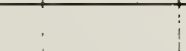 & 146 & 22 & 23 \\
\hline
\end{tabular}

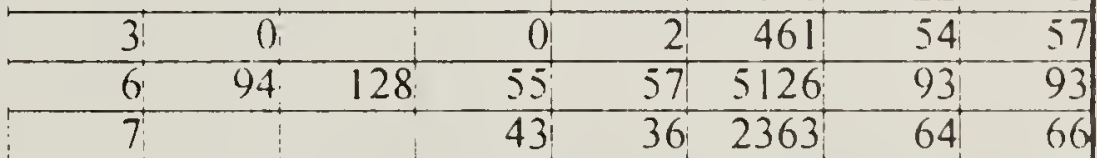

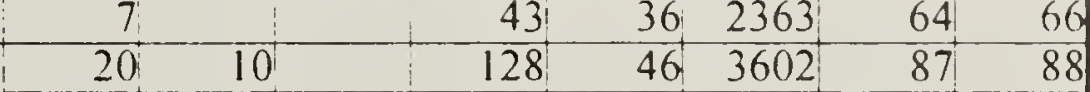

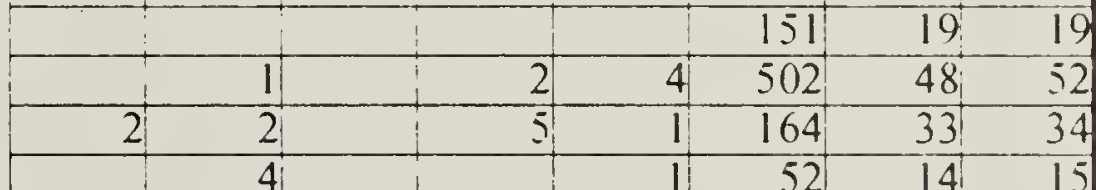

INDIVIDUALS ON COUNT DAY

SPECIES ON COUNT DAY

SPECIES COUNT PERIOD

INDIVIDUALS IN TABLES $4 \&$

SPECIES IN TABLES $4 \& 5$

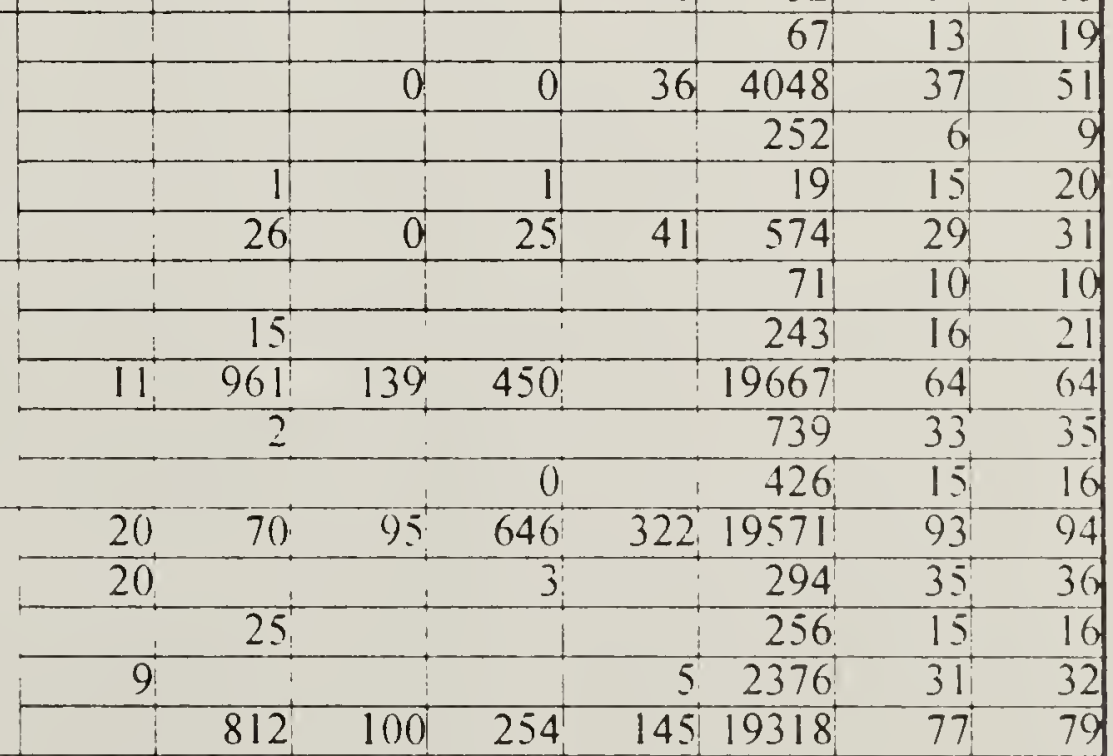


Table 4 - 1. SPECIES SEEN IN NINE OR FEWER LOCATIONS

\begin{tabular}{|c|c|}
\hline SPECIES & LOCATION AND NUMBERS \\
\hline WESTERN GREBE & Coronach, 1. \\
\hline AMERICAN WHITE PELICAN & Swift Current, + . \\
\hline DOUBLE-CRESTED CORMORANT & Gardiner Dam, 1. \\
\hline SNOW GOOSE & Coronach, 1; Fort Qu'Appelle, 1; Raymore, +; Weyburn, 4. \\
\hline GREEN-WINGED TEAL & Crooked Lake, 2; Estevan, I; Govenlock, 1. \\
\hline AMERICAN BLACK DUCK & Coronach, 1; Round Lake, ${ }^{+}$. \\
\hline NORTHERN PINTAIL & $\begin{array}{l}\text { Fort Qu'Appelle, 1; Gardiner Dam, 1; Saskatchewan } \\
\text { Landing Provincial Park, } 1 \text {. }\end{array}$ \\
\hline GADWALL & Crooked Lake, 1 \\
\hline CANVASBACK & Round Lake, 1; Saskatoon, 1. \\
\hline REDHEAD & $\begin{array}{l}\text { Estevan, 2; Fort Qu'Appelle, 1; Gardiner Dam, 15; Indian } \\
\text { Head, } 2 \text {. }\end{array}$ \\
\hline LESSER SCAUP & $\begin{array}{l}\text { Crooked Lake, 6; Estevan, 4; Fort Qu'Appelle, +; Gardiner } \\
\text { Dam, 5; Indian Head, } 1 \text {; Raymore, }+ \text {.Whitewood, }+.\end{array}$ \\
\hline BUFFLEHEAD & $\begin{array}{l}\text { Coronach, } 1 \text {; Crooked Lake, } 1 \text {; Fife Lake, } 1 \text {; Gardiner Dam } \\
2 \text {; Grayson, }+ \text {; Round Lake, 1; Squaw Rapids, } 1 .\end{array}$ \\
\hline HOODED MERGANSER & Fort Qu'Appelle, 2; Saskatoon, +. \\
\hline COMMON MERGANSER & $\begin{array}{l}\text { Coronach, 2; Crooked Lake, 1; Estevan, 15; Fort } \\
\text { Qu'Appelle, 1; Gardiner Dam, 207; Grayson, +; Round } \\
\text { Lake, 1; Squaw Rapids, } 8 .\end{array}$ \\
\hline NORTHERN HARRIER & $\begin{array}{l}\text { Clark's Crossing, +; Coronach, 1; Craven, 1; Estevan, 1; } \\
\text { Ferland, 1; Gardiner Dam, 1; Govenlock, 1; Rockglen, +. }\end{array}$ \\
\hline COOPER'S HAWK & Pike Lake. 1. \\
\hline RED-TAILED HAWK & Coronach, 1; Gardiner Dam, 1; Kenaston, 1; Saskatoon, 1. \\
\hline HARLAN'S HAWK & Fort Walsh, 1. \\
\hline FERRUGINOUS HAWK & Leader (south), 1. \\
\hline AMERICAN KESTREL & Regina, 1; Whitewood, +. \\
\hline PEREGRINE FALCON & Moose Jaw, + \\
\hline GYRFALCON & $\begin{array}{l}\text { Fort Qu'Appelle, +; Leader (south), 1; Pike Lake, 1; Skull } \\
\text { Creek, l. }\end{array}$ \\
\hline CHUKAR & Spalding, + . \\
\hline SPRUCE GROUSE & $\begin{array}{l}\text { Beauval, 2; Squaw Rapids, 25; Torch River Valley, +; } \\
\text { Turtle Lake, } 3 \text {. }\end{array}$ \\
\hline SAGE GROUSE & Govenlock, 2. \\
\hline WILD TURKEY & $\begin{array}{l}\text { Cabri, 14; Cypress Hills Provincial Park, 5; Estuary, 5; Fort } \\
\text { Walsh. } 2 \text {; Kennedy, } 11 \text {; MacDowall, } 1 .\end{array}$ \\
\hline AMERICAN COOT & Coronach, 23: Estevan, 23. \\
\hline K'ILILDEER & Govenlock. 1. \\
\hline COMMON SNIPE & Fort Walsh, 1: Saskatchewan Landing Provincial Park, 1. \\
\hline RING-BILLED GULL & Coronach, 1 . \\
\hline HERRING GULL & Gardiner Dam, 1. \\
\hline EURASIAN COLLARED-DOVE & Regina, 2 ; Weyburn, 2. \\
\hline MOURNING DOVE & Gardiner Dam, 1; Leader (north), +; Weyburn, 2. \\
\hline NORTHERN HAWK-OWL & Moose Jaw, I; Snowden, +; Squaw Rapids, 2. \\
\hline BARRED OWL & Love, 1; Squaw Rapids, 1; Stockholm, +. \\
\hline GREAT GRAY OWL & $\begin{array}{l}\text { Beauval, } 2 \text {; Nisbet Forest, +; Paddockwood, } 1 \text {; Prince } \\
\text { Albert, } 1 \text {; Turtle Lake, } 2 \text {. }\end{array}$ \\
\hline BOREAL OWL & Snowden, 1 \\
\hline NORTHERN SAW-WHET OWL & Livelong,.+ \\
\hline THREE-TOED WOODPECKER & Emma Lake, +; Squaw Rapids, 4. \\
\hline BLACK-BACKED WOODPECKER & Beauval, 2: Squaw Rapids, 1. \\
\hline NORTHERN FLICKER (Y-S) & $\begin{array}{l}\text { Estuary, 2; Fort Qu'Appelle, +; Grasslands National Park, } \\
\text { 1; Kindersley, +; Last Mountain Lake WMU, 1; Moose } \\
\text { Jaw, 2; Regina, 6: Saskatchewan Landing Provincial Park, } \\
\text { 2: Saskatoon. 26. }\end{array}$ \\
\hline
\end{tabular}


Table 4 - 2. SPECIES SEEN IN NINE OR FEWER LOCATIONS

\begin{tabular}{|c|c|}
\hline SPECIES & LOCATION AND NUMBERS \\
\hline NORTHERN FLICKER (R-S) & Saskatoon, + . \\
\hline NORTHERN FLICKER (YSXRS) & Saskatoon, 1 \\
\hline & Coronach, 1; Fort Walsh, 2; Moose Jaw, 1; Moose \\
\hline AMERICAN CROW & $\begin{array}{l}\text { Mountain, 1; Pike Lake, 1; Regina, 5; Saskatoon, 16; } \\
\text { Weyburn, 1. }\end{array}$ \\
\hline BROWN CREEPER & $\begin{array}{l}\text { Craven, +; Fort Walsh, 2; Kenosee Lake, 1; Saskatoon, +; } \\
\text { Swift Current, +. }\end{array}$ \\
\hline WINTER WREN & Fort Walsh, 1. \\
\hline VARIED THRUSH & Waldheim, + . \\
\hline BROWN THRASHER & Snowden, 1. \\
\hline RUFOUS-SIDED TOWHEE & Turtle Lake, 1. \\
\hline CHIPPING SPARROW & Watson. 3 \\
\hline FIELD SPARROW & Birch Hills. 1. \\
\hline SONG SPARROW & Crooked Lake, 1; Saskiatoon, + . \\
\hline WHITE-THROATED SPARROW & Love. 1; Whitewood, + . \\
\hline WHITE-CROWNED SPARROW & Craven, 2. \\
\hline HARRIS' SPARROW & Bangor, 3; Fort Walsh, 1; Snowden, +. \\
\hline DARK-EYED JUNCO (ORG) & Regina, 1. \\
\hline LAPLAND LONGSPUR & $\begin{array}{l}\text { Bromhead, 915; Coronach, 24: Govenlock, 22; Moose } \\
\text { Mountain, 42; Rockglen, 220; Weyburn, } 30 .\end{array}$ \\
\hline RED-WINGED BLACKBIRD & $\begin{array}{l}\text { Fort Walsh, 1; Regina, 33; Saskatchewan Landing } \\
\text { Provincial Park, } 1 .\end{array}$ \\
\hline WESTERN MEADOWLARK & Coronach, 2 ; Fort Qu'Appelle, ${ }^{+}$. \\
\hline RUSTY BLACKBIRD & $\begin{array}{l}\text { Clark's Crossing, 1: Coronach, 1; Estevan, 6; Raymore. +; } \\
\text { Yorkton, } 6 .\end{array}$ \\
\hline BREWER'S BLACKBIRD & Archerwill, 1; Bangor, 1; Estevan, 2. \\
\hline COMMON GRACKLE & $\begin{array}{l}\text { Beauval, 1; Birch Hills, 1; Fort Qu'Appelle, 1; Moose Jaw, } \\
\text { 2; Shamrock. } 2 .\end{array}$ \\
\hline BROWN-HEADED COWBIRD & Saskatoon. 1 \\
\hline BRAMBLING & MacDowall. ${ }^{+}$. \\
\hline GRAY-CROWNED ROSY FINCH & Coronach, $7 ;$ Fort Walsh, 3. \\
\hline PURPLE FINCH & $\begin{array}{l}\text { Fort Qu'Appelle. 6; Indian Head, 1: Kenosee Lake. 5; Pike } \\
\text { Lake. 1; Prince Albert National Park, 10; Round Lake. 2; } \\
\text { Saskatoon, 2; Swift Current, 18: Watson, } 4 \text {. }\end{array}$ \\
\hline RED CROSSBILL & $\begin{array}{l}\text { Cypress Hills Provincial Park, 3; Fort Walsh, 47; Squaw } \\
\text { Rapids, } 10 .\end{array}$ \\
\hline WHITE-WINGED CROSSBILL & $\begin{array}{l}\text { Beauval, 10; Candle Lake, 3; Fort Walsh, 3; Indian Head, } \\
\text { 4; Raymore, +; Saskatoon, 10; Squaw Rapids, } 19 .\end{array}$ \\
\hline AMERICAN GO & Craven, 10: Estevan, $4:$ Regina, $4:$ Yorkton, 1. \\
\hline
\end{tabular}

Table 5. BIRDS NOT IDENTIFIED TO SPECIES

\begin{tabular}{|c|c|}
\hline STECIES & ILOCATION ANT NOMRERS \\
\hline SMALL ACCIPITER species & Saskatoon. 1 \\
\hline BUTEO species & Fort Walsh, 2: Qu Appelle Valley Dam, 2: Shamrock. 1. \\
\hline EAGLE species & Saskatoon, 1. \\
\hline WOODPECKER species & Prince Albert National Park, I: Squaw Rapids. 5. \\
\hline BLACKBIRD species & Biggar, 1. \\
\hline CARPODACUS species & Govenlock, 1. \\
\hline CROSSBILL species & Squaw Rapids, 7. \\
\hline REDPOLL species & $\begin{array}{l}\text { Govenlock, 3; Prince Albert National Park, } 11 \text {; Squaw } \\
\text { Rapids. 31. }\end{array}$ \\
\hline
\end{tabular}


Table 6: NEW AND TYING 1999 HIGH COUNTS (new highs in bold italics)

\begin{tabular}{|c|c|c|c|c|}
\hline LOCATION & $\begin{array}{c}1999 \\
\text { COUNT }\end{array}$ & SPECIES & $\begin{array}{l}\text { PREV. } \\
\text { HIGH }\end{array}$ & $\begin{array}{l}\text { LOCATION } \\
\text { (YEAR) }\end{array}$ \\
\hline Coronach & 1 & \multirow{3}{*}{$\begin{array}{l}\text { WESTERN GREBE } \\
\text { DOUBLE-CREST CORMORANT } \\
\text { SNOW GOOSE }\end{array}$} & 1 & N. to Gardiner Dam \\
\hline Gardiner Dam & 1 & & 1 & N. to Squaw Rapids \\
\hline Weyburn & 4 & & 3 & Coronach 97 \\
\hline Gardiner Dam & 15 & \multirow{2}{*}{$\begin{array}{l}R E D H E A D \\
\text { COOPER'S HAWK }\end{array}$} & 8 & Regina 69 \\
\hline Pike Lake & 1 & & 1 & N. to Kamsack \\
\hline Fort Walsh & 1 & \multirow{5}{*}{$\begin{array}{l}\text { HARLAN'S HAWK } \\
\text { FERRUGINOUS HAWK } \\
\text { EURASIAN COLLARED-DOVE } \\
\text { BOREAL OWL } \\
\text { NORTHERN FLICKER (Y-S) }\end{array}$} & I & N. to White Bear \\
\hline Leader & $I$ & & New & \\
\hline Regina, Weyburn & 2 & & New & \\
\hline Snowden & 1 & & 1 & S. to Broadview \\
\hline Siskatoon & 26 & & 25 & Saskatoon 95 \\
\hline Fort Walsh & $I$ & \multirow{5}{*}{$\begin{array}{l}\text { WINTER WREN } \\
\text { BROWN THRASHER } \\
\text { RUFOUS-SIDED TOWHEE } \\
\text { CHIPPING SPARROW } \\
\text { FIELD SPARROW }\end{array}$} & New & \\
\hline Snowden & 1 & & 1 & Saskatoon 60 \\
\hline Turtle Lake & 1 & & 1 & N. to Leader \\
\hline Watson & 3 & & 2 & Swift Current 93 \\
\hline Birch Hills & 1 & & 1 & Saskatoon CP 85 \\
\hline Craven & 2 & \multirow{4}{*}{$\begin{array}{l}\text { WHITE-CROWNED SPARROW } \\
\text { BRAMBLING } \\
\text { HOUSE FINCH } \\
\text { AMERICAN GOLDFINCH }\end{array}$} & $I$ & N. to Saskatoon \\
\hline MacDowall & $C P$ & & New & \\
\hline Saskatoon & 247 & & 190 & Regina 98 \\
\hline Craven & 10 & & 9 & Kenosee Lake 98 \\
\hline
\end{tabular}

Table 7: POPULATION STATUS OF SELECTED SPECIES ( birds/hour effort)

\begin{tabular}{|c|c|c|c|c|c|}
\hline SPECIES & $\begin{array}{l}n \\
2 \\
\sigma \\
\sigma\end{array}$ & 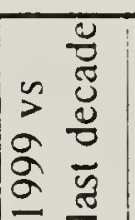 & SPECIES & $\begin{array}{ll}\infty & \\
2 & 0 \\
2 & \infty \\
2 & 2\end{array}$ & 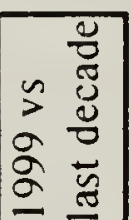 \\
\hline \multirow{5}{*}{$\begin{array}{l}\text { CANADA GOOSE } \\
\text { MALLARD } \\
\text { COMMON GOLDENEYE } \\
\text { BALD EAGLE } \\
\text { NORTHERN GOSHAWK }\end{array}$} & 273 & $\overline{80}$ & \multirow{5}{*}{$\begin{array}{l}\text { COMMON RAVEN } \\
\text { BLACK-CAPPED CHICKADEE } \\
\text { BOREAL CHICKADEE } \\
\text { RED-BREASTED NUTHATCH } \\
\text { WHITE-BREAST NUTHATCH }\end{array}$} & -13 & $\overline{28}$ \\
\hline & 58 & 136 & & -20 & 9 \\
\hline & 19 & 5 & & -3 & 25 \\
\hline & 55 & 27 & & 107 & 57 \\
\hline & -2 & -26 & & -17 & 2 \\
\hline \multirow{5}{*}{$\begin{array}{l}\text { GOLDEN EAGLE } \\
\text { MERLIN } \\
\text { PRAIRIE FALCON } \\
\text { GRAY PARTRIDGE } \\
\text { RING-NECKED PHFASANT }\end{array}$} & 9 & -16 & \multirow{5}{*}{$\begin{array}{l}\text { BROWN CREEPER } \\
\text { GOLDEN-CROWNED KINGLET } \\
\text { AMERICAN ROBIN } \\
\text { BOHEMIAN WAXWING } \\
\text { CEDAR WAXWING }\end{array}$} & -68 & -55 \\
\hline & -45 & -26 & & 77 & 65 \\
\hline & 70 & 44 & & 128 & -19 \\
\hline & -35 & -47 & & -80 & -59 \\
\hline & -62 & -57 & & 198 & -51 \\
\hline SPRUCE GROUSE & 155 & 191 & \multirow{5}{*}{$\begin{array}{l}\text { NORTHERN SHRIKE } \\
\text { EUROPEAN STARLING } \\
\text { AMERICAN TREE SPARROW } \\
\text { DARK-EYED JUNCO } \\
\text { LAPLAND LONGSPUR }\end{array}$} & 1 & -27 \\
\hline RUFFED GROUSE & -14 & 8 & & 41 & 53 \\
\hline SHARP-TAILED GROUSE & 2 & -6 & & 63 & 125 \\
\hline \multirow{2}{*}{$\begin{array}{l}\text { ROCK DOVE } \\
\text { GREAT HORNED OWL }\end{array}$} & 1 & 0 & & 43 & 48 \\
\hline & -13 & -25 & & -31 & -30 \\
\hline SNOWY OWL & 0 & -33 & \multirow{5}{*}{$\begin{array}{l}\text { SNOW BUNTING } \\
\text { RUSTY BLACKBIRD } \\
\text { PINE GROSBEAK } \\
\text { HOUSE FINCH } \\
\text { RED CROSSBILL }\end{array}$} & -32 & -11 \\
\hline \multirow{4}{*}{$\begin{array}{l}\text { SHORT-EARED OWL } \\
\text { DOWNY WOODDPECKER } \\
\text { HAIRY WOODPECKER } \\
\text { THREE-TOE WOODPECKER }\end{array}$} & 3 & 11 & & -8 & -58 \\
\hline & -19 & 5 & & -33 & -47 \\
\hline & -21 & 1 & & -7 & 283 \\
\hline & -57 & -55 & & -68 & -27 \\
\hline \multirow{4}{*}{$\begin{array}{l}\text { NORTHERN FLICKER } \\
\text { PILEATED WOODPECKER } \\
\text { HORNED LARK } \\
\text { GRAY JAY }\end{array}$} & 4 & 18 & \multirow{2}{*}{$\begin{array}{l}\text { WHITE-WINGED CROSSBILL } \\
\text { COMMON REDPOLL }\end{array}$} & -3 & -86 \\
\hline & 48 & 43 & & 322 & 316 \\
\hline & -72 & -53 & \multirow{4}{*}{$\begin{array}{l}\text { HOARY REDPOLL } \\
\text { PINE SISKIN } \\
\text { EVENING GROSBEAK } \\
\text { HOUSE SPARROW }\end{array}$} & 233 & 149 \\
\hline & -7 & 3 & & -40 & 51 \\
\hline BLUE JAY & -45 & -24 & & 142 & \\
\hline BLACK-BILLED MAGPIE & -17 & $\sqrt{18}$ & & -25 & \\
\hline
\end{tabular}

